\title{
LINEAR SOFIC GROUPS AND ALGEBRAS
}

\author{
GOULNARA ARZHANTSEVA AND LIVIU PĂUNESCU
}

\begin{abstract}
We introduce and systematically study linear sofic groups and linear sofic algebras. This generalizes amenable and LEF groups and algebras. We prove that a group is linear sofic if and only if its group algebra is linear sofic. We show that linear soficity for groups is a priori weaker than soficity but stronger than weak soficity. We also provide an alternative proof of a result of Elek and Szabo which states that sofic groups satisfy Kaplansky's direct finiteness conjecture.
\end{abstract}

\section{INTRODUCTION}

Metric approximation properties for groups have received considerable attention in the last years, mainly due to the notions of hyperlinear and sofic groups. Hyperlinear groups appeared in the context of Alain Connes' embedding conjecture (1976) in operator algebra and were introduced by Florin Rădulescu [Ră08]. Sofic groups were introduced by Misha Gromov [Gr99] in his study of symbolic algebraic varieties in relation to the Gottschalk surjunctivity conjecture (1973) in topological dynamics. They were called sofic by Weiss [W00]. Over the last years, various strong results have been obtained for sofic groups in seemingly unrelated areas of mathematics. For instance, they have been at the heart of developments on profinite topology of free groups, unimodular random networks, diophantine approximations, linear cellular automata, $L^{2}$-torsion, profinite equivalence relations, measure conjugacy invariants, and continuous (in contrast to traditional binary) logic.

These group properties can be stated in elementary algebraic terms, as approximation properties, or in the language of ultraproducts, as the existence of an embedding in

2010 Mathematics Subject Classification. 20E26, 20C07, 16N99, 03C20, 20 F70.

Key words and phrases. Sofic groups, metric ultraproduct, linear groups, Kaplansky's direct finiteness conjecture.

The first author was supported in part by the ERC grant ANALYTIC no. 259527, and by the Swiss NSF, under Sinergia grant CRSI22-130435. The second author was supported by the Swiss NSF, under Sinergia grant CRSI22-130435. 
a certain metric ultraproduct. We mainly use the later technique due to simplicity in writing. For a careful introduction to the subject, including ultraproducts terminology, see [Pe08, PeKw09].

Throughout the article, let $\omega$ be a non-principal (or free) ultrafilter on $\mathbb{N}$. In general, $\left(n_{k}\right)_{k}$ or $\left(m_{k}\right)_{k}$ denote sequences of natural numbers tending to infinity. We denote by $S_{n}$ the symmetric group of degree $n$, that is the group of permutations on a set of $n$ elements. This group is endowed with the normalized Hamming distance:

$$
d_{\text {Hamm }}(p, q)=\frac{1}{n}|\{i: p(i) \neq q(i)\}| .
$$

Definition 1.1. A group $G$ is sofic if there exist a sequence of natural numbers $\left(n_{k}\right)_{k}$ and an injective group morphism from $G$ into the metric ultraproduct $\prod_{k \rightarrow \omega}\left(S_{n_{k}}, d_{H a m m}\right)$.

Such a morphism is called a sofic representation of $G$.

The goal of our paper is to introduce soficity for algebras. We shall approximate our algebras by matrix algebras endowed with a distance provided by the rank. Two matrices are close in this distance if they are equal, as linear transformations, on a large subspace. This is in essence similar to the Hamming distance. Therefore, we call the corresponding algebras (and groups, respectively) linear sofic. We refer the reader to Section 4 for precise definitions.

Our main results about linear soficity are the following.

Theorem 1.2. A group $G$ is linear sofic if and only if its group algebra $\mathbb{C} G$ is linear sofic.

This has to be regarded in the light of recent developments in asymptotic geometry of algebras and, more specifically, of group algebras, see [Gr08, CS09] and references therein. In particular, the known fact that a group is amenable if and only if its group algebra is amenable [S00, El05, B08] is an evident predecessor of the above result.

Our proof of this theorem also provides an alternative proof of Kaplansky's direct finiteness conjecture for sofic groups, a result due to Elek and Szabo [ElSz04].

In [G1Ri08] Glebsky and Rivera defined the notion of weakly sofic group by replacing $\left(S_{n}, d_{\text {Hamm }}\right)$ in the definition of sofic groups by arbitrary finite groups equipped with a bi-invariant metric. At present, quite a few is known about weakly sofic groups.

Theorem 1.3. Sofic groups are linear sofic, while linear sofic groups are weakly sofic. 
Our viewpoint on the approximation of algebras and groups has given rise to a number of challenging difficulties. For instance, the equivalence between the metric ultraproduct interpretation and the algebraic approach in the definition of approximation, as well as the fundamental amplification trick, are easy in the sofic and hyperlinear cases. In the rank metric case, both properties are highly non-trivial. We successfully resolve these issues by introducing the rank amplification and by analyzing the tensor product of Jordan blocks, see Sections 4 and 5 .

Our approach leads to interesting phenomena (nonexistent in the classical sofic case) when approximations by complex matrix algebras are replaced by those over a different field (or a sequence of fields), see Sections 6 and 7, For instance, using the fundamental result of real semialgebraic geometry, so-called Positivstellensat 1 , we establish the equivalence between linear sofic representations over the field of complex numbers and those over the rationals.

Although Kaplansky's direct finiteness conjecture remains open for linear sofic groups (see Questions 7.9 and 8.6), we show that this new class of groups share with sofic groups several positive results. In particular, the class of linear sofic groups is preserved under many group-theoretical operations, see Section 9 . Moreover, under the failure of the Continuum Hypothesis, there exist $2^{\aleph_{c}}$ of universal linear sofic groups, up to metric isomorphism, see Section 10 .

Our intention to study soficity of algebras is motivated by recent advances on amenable algebras as well as LEF algebras and algebras having almost finite dimensional representations [VG97, $\mathrm{Zi02}, \mathrm{El03}, \mathrm{El05}, \mathrm{Gr} 08]$ ]. The idea allows to go beyond algebras associated to groups. In such a general context, we introduce the concepts of linear sofic radical for groups and of sofic radical for algebras. We also notice the existence of algebras which are not linear sofic (these are not group algebras). We refer to Section 11 for details.

Our choice of the rank metric is not arbitrary but has a view towards potential applications. The concept of the rank metric was first introduced by Loo-Keng Hua [Hua45] (he uses the term "arithmetic distance") who found a surprisingly nice description of adjacency preserving maps with respect to this metric. The entire book [Wan96] is devoted to this topic, see also [S06] for a recent discussion on the connection to several preserver problems on matrix and operator algebras arising in physics and

\footnotetext{
${ }^{1}$ This is a semialgebraic analogue of famous Hilbert's Nullstellensatz.
} 
geometry. From a different point of view, Philippe Delsarte [Del78] defined the rank distance (named $q$-distance) on the set of bilinear forms and proposed the construction of optimal codes in bilinear form representation. This allowed Ernst Gabidulin [Gab85] to study the rank distance for vector spaces over extension fields and to describe optimal codes, now called Gabidulin codes. This currently emerged into an intensively developing area of rank-metric codes.

In view of the above, we believe that the full extent of possible applications of sofic and linear sofic groups and algebras is yet to be discovered. The present paper provides the necessary fundaments for such further developments.

\section{ULTRAPRODUCTS OF MATRIX ALGEBRAS WITH RESPECT TO THE RANK}

Ultraproducts of matrices using rank functions have been considered, for example, in [ElSz04, Oz09]. Let us first recall some basic properties of the rank. Throughout the article $F$ is an arbitrary field.

Notation 2.1. For a matrix $a \in M_{n}=M_{n}(F)$ we shall denote by $r k(a)$ its rank and define the normalized rank by $\rho(a):=\frac{1}{n} r k(a)$.

Proposition 2.2. The rank function on complex matrices has the following properties:

(1) $r k\left(I_{n}\right)=n ; r k(a)=0$ if and only if $a=0$;

(2) $r k(u+v) \leqslant r k(u)+r k(v)$ for $u, v \in M_{n}$;

(3) $r k(u v) \leqslant r k(u)$ and $r k(u v) \leqslant r k(v)$ for $u, v \in M_{n}$;

(4) $r k(u \oplus v)=r k(u)+r k(v)$ for $u \in M_{n}$ and $v \in M_{m}$;

(5) $r k(u \otimes v)=r k(u) \cdot r k(v)$ for $u \in M_{n}$ and $v \in M_{m}$.

We now define the ultraproduct that we use throughout the paper.

Definition 2.3. Let $\omega$ be a non-principal (or free) ultrafilter and $\left(n_{k}\right)_{k}$ a sequence of natural numbers such that $\lim _{k \rightarrow \infty} n_{k}=\infty$. The Cartesian product $\Pi M_{n_{k}}(F)$ is an algebra. Let us define:

$$
\rho_{\omega}: \Pi M_{n_{k}}(F) \rightarrow[0,1] \quad \rho_{\omega}\left(\left(a_{k}\right)_{k}\right):=\lim _{k \rightarrow \omega} \rho\left(a_{k}\right) .
$$

Then $K \operatorname{Ker} \rho_{\omega}$ is an ideal of $\Pi M_{n_{k}}(F)$. We denote by $\Pi_{k \rightarrow \omega} M_{n_{k}}(F) / K e r \rho_{\omega}$, or by $\Pi M_{n_{k}}(F) / \operatorname{Ker} \rho_{\omega}$ if there is no danger of confusion, the ultraproduct obtained by taking the quotient of $\Pi M_{n_{k}}(F)$ by $K \operatorname{Ker} \rho_{\omega}$. This algebra comes with a natural metric defined by $d_{\omega}(a, b):=\rho_{\omega}(a-b)$, where $a$ and $b$ belong to the ultraproduct. 
We always denote by $\rho_{\omega}$ the limit rank function, even though we shall work with ultraproducts over different dimension sequences $\left(n_{k}\right)_{k}$. It will be clear what is the dimension of the matrices that we use, so this notation should cause no confusion.

Observation 2.4. The function $\rho$ induces a metric on $M_{n}(F)$, defined by $d_{r k}(a, b):=$ $\rho(a-b)$. This metric restricted to the group $G L_{n}(F)$ is bi-invariant. Thus, we can construct the following ultraproduct.

Definition 2.5. We denote by $\prod_{k \rightarrow \omega} G L_{n_{k}}(F) / d_{\omega}$ the metric ultraproduct obtained by taking the quotient of the Cartesian product $\Pi G L_{n_{k}}(F)$ by $\mathcal{N}_{\omega}=\left\{\left(a_{k}\right)_{k} \in \Pi G L_{n_{k}}(F)\right.$ : $\left.\lim _{k \rightarrow \omega} d_{r k}\left(a_{k}, I d\right)=0\right\}$.

In many ultraproduct constructions invertible elements in an ultraproduct are given by ultraproduct of invertible elements. For instance, this is the case in the classical result of Malcev addressing the algebraic ultraproduct of matrix algebras [Ma40]. An analogous result holds also for our rank ultraproduct construction.

Proposition 2.6. The group $\mathcal{U}\left(\Pi_{k \rightarrow \omega} M_{n_{k}}(F) / \operatorname{Ker} \rho_{\omega}\right)$ of invertible elements of $\Pi_{k \rightarrow \omega} M_{n_{k}}(F) /$ Ker $\rho_{\omega}$ is isomorphic to $\prod_{k \rightarrow \omega} G L_{n_{k}}(F) / d_{\omega}$.

Proof. Elements of $\prod_{k \rightarrow \omega} G L_{n_{k}}(F) / d_{\omega}$ are invertible. So, $\prod_{k \rightarrow \omega} G L_{n_{k}}(F) / d_{\omega} \subseteq$ $\mathcal{U}\left(\Pi_{k \rightarrow \omega} M_{n_{k}}(F) / K e r \rho_{\omega}\right)$. For the converse inclusion, the key observation is that for any $a \in M_{n_{k}}(F)$ there exists $\tilde{a} \in G L_{n_{k}}(F)$ such that $\rho(a-\tilde{a})=1-\rho(a)$.

If $\left(a_{k}\right)_{k, \omega}$ is invertible in $\Pi_{k \rightarrow \omega} M_{n_{k}}(F) / \operatorname{Ker} \rho_{\omega}$ then $\rho_{\omega}\left(\left(a_{k}\right)_{k, \omega}\right)=1$ by (1) and (3) of Proposition 2.2 (also the reverse of this implication holds). It follows that $\left(a_{k}\right)_{k, \omega}=\left(\tilde{a}_{k}\right)_{k, \omega} \in \prod_{k \rightarrow \omega} G L_{n_{k}}(F) / d_{\omega}$.

We shall encounter many examples of stably finite algebras. Let us first recall the definition.

Definition 2.7. A unital ring $R$ is called directly finite if for any $x, y \in R, x y=I$ implies $y x=I$. It is called stably finite if $M_{n}(R)$ is directly finite for any $n \in \mathbb{N}$.

Kaplansky's Direct Finiteness Conjecture. For any field $F$ and any countable group $G$ the group algebra $F(G)$ is directly finite.

The following proposition is well known. It was used by Elek and Szabo to prove Kaplansky's direct finiteness conjecture for sofic groups and it also appears in [Oz09]. Note that the class of sofic goups is currently the largest known to satisfy this conjecture. 
Proposition 2.8. The algebra $\prod_{k \rightarrow \omega} M_{n_{k}}(F) / K \operatorname{Ker} \rho_{\omega}$ is stably finite.

Proof. As $M_{m}\left(\Pi_{k \rightarrow \omega} M_{n_{k}}(F) / \operatorname{Ker} \rho_{\omega}\right) \simeq \Pi_{k \rightarrow \omega} M_{m \cdot n_{k}}(F) / \operatorname{Ker} \rho_{\omega}$ we only need to prove the direct finiteness of these algebras.

It is not hard to check that $r k(I-a b)=r k(I-b a)$ for $a, b \in M_{n}(F)$. This equality implies in the ultralimit that $\rho_{\omega}(I-x y)=\rho_{\omega}(I-y x)$. So, in $\Pi_{k \rightarrow \omega} M_{n_{k}}(F) / \operatorname{Ker} \rho_{\omega}$ we have $x y=I$ if and only if $y x=I$.

\section{PROduct of Ultrafilters}

We have equipped the ultraproduct $\prod_{k \rightarrow \omega} M_{n_{k}}(F) / K \operatorname{Ker} \rho_{\omega}$ with a metric induced by the rank function, namely $d_{\omega}(a, b):=\rho_{\omega}(a-b)$. If we have a family of ultraproducts we can construct the metric ultraproduct of this family. The object that we get is again an ultraproduct. We provide here the definitions. For more details, we refer the reader to [CaPă12].

Definition 3.1. If $\phi, \omega$ are ultrafilters on $\mathbb{N}$, then define the product ultrafilter $\phi \otimes \omega$ on $\mathbb{N} \times \mathbb{N}$ by:

$$
A \in \phi \otimes \omega \Longleftrightarrow\{i \in \mathbb{N}:\{j \in \mathbb{N}:(i, j) \in A\} \in \omega\} \in \phi .
$$

It is easy to check that $\phi \otimes \omega$ is an ultrafilter. Since $\mathbb{N}$ and $\mathbb{N}^{2}$ are cardinal equivalent, $\phi \otimes \omega$ can be viewed as an ultrafilter on $\mathbb{N}$.

Proposition 3.2. If $\left(x_{i}^{j}\right)_{(i, j) \in \mathbb{N}^{2}}$ is a bounded sequence of real numbers then:

$$
\lim _{i \rightarrow \phi}\left(\lim _{j \rightarrow \omega} x_{i}^{j}\right)=\lim _{(i, j) \rightarrow \phi \otimes \omega} x_{i}^{j} .
$$

This proposition implies that the ultraproduct of ultraproducts is again an ultraproduct:

Corollary 3.3. Let $\left(n_{m, k}\right)_{m, k}$ be a double sequence of natural numbers. For every $m \in \mathbb{N}$ construct the ultraproduct $\Pi_{k \rightarrow \omega} M_{n_{m, k}} / \operatorname{Ker} \rho_{\omega}$. On the Cartesian product $\Pi_{m}\left(\Pi_{k \rightarrow \omega} M_{n_{k, m}}\right)$ define $d_{\phi}\left(\left(a_{m}\right)_{m},\left(b_{m}\right)_{m}\right)=\lim _{m \rightarrow \phi} \rho_{\omega}\left(a_{m}-b_{m}\right)$. Then:

$$
\Pi_{m \rightarrow \phi}\left(\Pi_{k \rightarrow \omega} M_{n_{m, k}} / \operatorname{Ker} \rho_{\omega}\right) / d_{\phi} \simeq \Pi_{(m, k) \rightarrow \phi \otimes \omega} M_{n_{m, k}} / \operatorname{Ker} \rho_{\phi \otimes \omega} .
$$




\section{DEFINITIONS OF LINEAR SOFICITY}

We are now defining the main concepts of our paper.

Definition 4.1. A countable group $G$ is linear sofic if there exist an injective morphism $\Theta: G \rightarrow \prod_{k \rightarrow \omega} G L_{n_{k}}(\mathbb{C}) / d_{\omega}$.

Such a morphism is called a linear sofic representation of $G$.

Definition 4.2. A countably generated algebra $A$ over a field $F$ is linear sofic if there exist an injective morphism $\Theta: A \rightarrow \prod_{k \rightarrow \omega} M_{n_{k}}(F) / \operatorname{Ker} \rho_{\omega}$. Moreover, if $A$ is a unital algebra we require that this morphism is unital.

Such a morphism is called a linear sofic representation of $A$.

Observation 4.3. An element of $M_{n}(\mathbb{C})$ is a linear transformation of the vector space $\mathbb{C}^{n}$. As $\mathbb{C}$ is a vector space of dimension 2 over $\mathbb{R}$, we can view this element as a transformation of the space $\mathbb{R}^{2 n}$ or as a matrix in $M_{2 n}(\mathbb{R})$. Its normalized rank remains the same. As a consequence, a morphism $\Theta: G \rightarrow \prod_{k \rightarrow \omega} G L_{n_{k}}(\mathbb{C}) / K e r \rho_{\omega}$ induces a morphism $\Theta^{\prime}: G \rightarrow \prod_{k \rightarrow \omega} G L_{2 n_{k}}(\mathbb{R}) / \operatorname{Ker} \rho_{\omega}$. The value of $\rho_{\omega}$ is preserved by this transformation. It follows that we can work with $\mathbb{R}$ instead of $\mathbb{C}$ in the definition of linear sofic group. We can further reduce our considerations to the field of rationals (or equivalently to any finite dimensional extension of the rationals), see Section 6. Alternatively, we construct linear sofic representations of groups over a sequence of finite fields, see Section 8 .

Let $G$ be a linear sofic group and $\Theta: G \rightarrow \prod_{k \rightarrow \omega} G L_{n_{k}} / d_{\omega}$ be an injective morphism. We define a length function $\delta: G \rightarrow[0,1]$ by $\delta(g)=d_{\omega}(1, \Theta(g))$. Then $\delta$ is constant on conjugacy classes of $G$ and $\delta(g)=0$ iff $g=e$. The following proposition is straightforward. It gives a more algebraic definition of linear soficity. We will provide a stronger version we alluded to in the introduction, see Section 5 ,

Proposition 4.4. A group $G$ is linear sofic if and only if the following holds: there exists $\delta: G \rightarrow[0,1]$ such that $\delta(g)=0$ if and only if $g=e$ and for any finite subset $E \subset G$ and for any $\varepsilon>0$, there exist $n \in \mathbb{N}$ and a function $\phi: E \rightarrow G L_{n}(\mathbb{C})$ such that:

(1) $\forall g, h, g h \in E$ we have $\rho(\phi(g) \phi(h)-\phi(g h))<\varepsilon$;

(2) $\forall g \in E$ we have $\rho(1-\phi(g))>\delta(g)-\varepsilon$. 
Using this equivalent characterization we see that the definition of linear sofic group does not depend on the particular choice of the ultrafilter nor does it depend on the sequence $\left(n_{k}\right)_{k}$ as long as $\lim _{k \rightarrow \infty} n_{k}=\infty$.

It is implicit in [ElSz04] that for a sofic group $G$ and any field $F$ the group algebra $F(G)$ satisfies our definition of linear sofic algebra (see also [0z09]). This was obtained as an intermediate result in the proof of Kaplansky's direct finiteness conjecture for sofic groups. We present an alternative proof of this fact in Section 7, as a consequence of our results about linear soficity. The following result shows that linear soficity is a priori weaker than soficity. Observe that the converse is open.

Proposition 4.5. Sofic groups are linear sofic.

Proof. Let $p \in S_{n}$ and let $A_{p}$ be the corresponding permutation matrix. Denote by $f i x(p)$ the number of fixed points of $p$ and by $c y c(p)$ the number of cycles (including fixed points) of $p$. Clearly, $f i x(p) \leqslant c y c(p)$. By definition, $d_{\text {Hamm }}(I d, p)=1-f i x(p) / n$ and it is easy to check that $\rho\left(I d-A_{p}\right)=1-\operatorname{cyc}(p) / n$ (see [Lu11], Lemma 13). From this we deduce that $\rho\left(I d-A_{p}\right) \leqslant d_{\text {Hamm }}(I d, p)$.

Let $G$ be a sofic group and $\Phi: G \rightarrow \prod_{k \rightarrow \omega}\left(S_{n_{k}}, d_{\text {Hamm }}\right)$ the corresponding injective morphism. The group $S_{n_{k}}$ is isomorphic to the subgroup of permutation matrices of $G L_{n_{k}}(\mathbb{C})$. Due to the above inequality on the normalized rank, the morphism $\Phi$ induces a group morphism $\Theta: G \rightarrow \prod_{k \rightarrow \omega} G L_{n_{k}}(\mathbb{C}) / d_{\omega}$. We show that this morphism is injective.

Coming back to $p$ and $A_{p}$, it is easy to see that $c y c(p) \leqslant f i x(p)+(n-f i x(p)) / 2$. This can be rewritten as $1-f i x(p) / n \leqslant 2(1-c y c(p) / n)$. Thus,

$$
d_{\text {Hamm }}(I d, p) \leqslant 2 \rho\left(I d-A_{p}\right) .
$$

As a consequence, we deduce the injectivity of $\Theta$.

\section{RANK AMPLIFICATION}

A classical theorem of Elek and Szabo states that if $G$ is a sofic group then there exists a group morphism $\Theta: G \rightarrow \prod_{k \rightarrow \omega}\left(S_{n_{k}}, d_{\text {Hamm }}\right)$ such that the distance between $\Theta\left(g_{1}\right)$ and $\Theta\left(g_{2}\right)$ is 1 in the limit for each $g_{1} \neq g_{2}$. This fact is required to prove various results including some permanence properties like a direct limit of sofic groups is again sofic. We shall obtain a similar general fact for linear sofic groups. That is, in Proposition 4.4 we make a function $\delta$ constant on $G \backslash\{e\}: \delta$ is independent of the choice of the group 
element $g \in G \backslash\{e\}$. The proof of Elek and Szabo employs a fundamental tool called amplification. In matrix language, this tool relies on tensor products together with the formula $\operatorname{Tr}(a \otimes b)=\operatorname{Tr}(a) \operatorname{Tr}(b)$, where $\operatorname{Tr}$ denotes the trace of a matrix. Unfortunately, we do not have a similar formula for the rank metric. Thus, our proof is technically much more involved.

5.1. Preliminaries. In this section, $A$ is always an element of $G L_{n}(\mathbb{C})$. For $\lambda \in \mathbb{C}$ define $M_{\lambda}(A)$ to be $1 / n$ multiplied with the algebraic multiplicity of the eigenvalue $\lambda$ (this is 0 whenever $\lambda$ is not an eigenvalue of $A$ ). Then $M_{0}(A)=0$ and $\sum_{\lambda \in \mathbb{C}^{*}} M_{\lambda}(A)=1$. Observe that:

$$
\rho(A-I d) \geqslant 1-M_{1}(A) .
$$

Lemma 5.1. If $\left(\lambda_{i}\right)_{i=1, \ldots, n}$ are the eigenvalues of $A$ written with the algebraic multiplicity, then $\left(\lambda_{i} \lambda_{j}\right)_{i, j=1, \ldots, n}$ are the eigenvalues of $A \otimes A$ written with algebraic multiplicity.

Proof. By the Jordan decomposition, we can write $A$ as an upper triangular matrix with the values $\left(\lambda_{i}\right)_{i=1, \ldots, n}$ on the diagonal. Then $A \otimes A$ is also an upper triangular matrix with the values $\left(\lambda_{i} \lambda_{j}\right)_{i, j=1, \ldots, n}$ on the diagonal. This implies that $\left(\lambda_{i} \lambda_{j}\right)_{i, j=1, \ldots, n}$ are the roots of the characteristic polynomial of $A \otimes A$. These roots are the eigenvalues with algebraic multiplicity.

Lemma 5.2. If $A \in G L_{n}(\mathbb{C})$, then $M_{1}(A \otimes A) \leqslant M_{1}(A)^{2}+\left(1-M_{1}(A)\right)^{2}$.

Proof. Let $\left(\lambda_{i}\right)_{i=1, \ldots, n}$ be the eigenvalues of $A$ written with the algebraic multiplicity. Assume that for $i=1, \ldots, k$ we have $\lambda_{i}=1$ and for $i=k+1, \ldots, n$ we have $\lambda_{i} \neq 1$. Then $M_{1}(A)=k / n$. If $\lambda_{i} \lambda_{j}=1$ then either $i \leqslant k$ and $j \leqslant k$ or $i>k$ and $j>k$. This implies that $M_{1}(A \otimes A) \leqslant\left(k^{2}+(n-k)^{2}\right) / n^{2}=M_{1}(A)^{2}+\left(1-M_{1}(A)\right)^{2}$.

The following proposition is elementary.

Proposition 5.3. Define $f:[1 / 2,1] \rightarrow[1 / 2,1]$ by $f(x)=x^{2}+(1-x)^{2}$. Then $f$ is $a$ well-defined increasing bijection. If $x \in[1 / 2,1)$, then $\lim _{m \rightarrow \infty} f^{m}(x)=1 / 2$.

Lemma 5.4. If there exists $\mu \in \mathbb{C}$ such that $M_{\mu}(A \otimes A)>1 / 2$, then there exists $\lambda \in \mathbb{C}$ such that $M_{\lambda}(A)>1 / 2$. Moreover, both $\lambda$ and $\mu$ are unique and if $\lambda=1$ then $\mu=1$.

Proof. Let $\left(\lambda_{i}\right)_{i=1, \ldots, n}$ be the eigenvalues of $A$ written with the algebraic multiplicity. By hypothesis, there exists $\mu \in \mathbb{C}$ such that $\left|\left\{(i, j): \lambda_{i} \lambda_{j}=\mu\right\}\right|>\frac{1}{2} n^{2}$. Let 
$C_{i}=\left\{j: \lambda_{i} \lambda_{j}=\mu\right\}$. Then $\sum_{i=1}^{n}\left|C_{i}\right|>\frac{1}{2} n^{2}$. It follows that there exists $i_{0}$ such that $\left|C_{i_{0}}\right|>\frac{1}{2} n$. If $\lambda=\lambda_{i_{0}}^{-1} \mu$ then $\lambda_{j}=\lambda$ for every $j \in C_{i_{0}}$. This means that $M_{\lambda}(A)>1 / 2$. The uniqueness part of the proposition is trivial.

Suppose now that $\lambda=1$ and assume that $\mu \neq 1$. Let $k_{1}=M_{1}(A) \cdot n$ and $k_{2}=M_{\mu}(A) \cdot n$ (these are the algebraic multiplicities of 1 and $\mu$ ). Define $k_{3}=n-k_{1}-k_{2}$. By hypothesis $k_{1}>n / 2$, hence $k_{3}<k_{1}$. It is easy to see that the algebraic multiplicity of $\mu$ in $A \otimes A$ is less than $2 k_{1} k_{2}+k_{3}^{2}$. Then:

$2 n^{2} M_{\mu}(A \otimes A) \leqslant 4 k_{1} k_{2}+2 k_{3}^{2} \leqslant\left(k_{1}+k_{2}\right)^{2}+\left(k_{1}+k_{2}\right) k_{3}+k_{3}^{2} \leqslant\left(k_{1}+k_{2}+k_{3}\right)^{2}=n^{2}$.

It follows that $M_{\mu}(A \otimes A) \leqslant 1 / 2$, giving a contradiction.

Definition 5.5. For $A \in G L_{n}(\mathbb{C})$ define $A_{1}:=A$ and $A_{m+1}:=A_{m} \otimes A_{m}$.

Proposition 5.6. Suppose that $M_{1}(A) \in(1 / 2,1)$ and let $c$ be a constant such that $c \in\left(M_{1}(A), 1\right)$. Then $M_{1}\left(A_{m}\right)<f^{m-1}(c)$.

Proof. We proceed by induction. The case $m=1$ follows by hypothesis. Assume that $M_{1}\left(A_{m}\right)<f^{m-1}(c)$. Since $f^{m}(c)$ is always strictly greater than $1 / 2$, if $M_{1}\left(A_{m+1}\right) \leqslant 1 / 2$ we are done. Assume that $M_{1}\left(A_{m+1}\right)>1 / 2$. We shall prove that also $M_{1}\left(A_{m}\right)>1 / 2$.

By the previous lemma and a reverse induction, for each $n \leqslant m$ there exists $\lambda_{n}$ such that $M_{\lambda_{n}}\left(A_{n}\right)>1 / 2$. Since $M_{1}(A)>1 / 2$ we get $\lambda_{1}=1$. Applying the second part of the previous lemma, we inductively get $\lambda_{n}=1$ for $n \leqslant m$. This proves that $M_{1}\left(A_{m}\right)>1 / 2$.

The function $f$ is strictly increasing, therefore $f\left(M_{1}\left(A_{m}\right)\right)<f^{m}(c)$. By Lemma 5.2, we get $M_{1}\left(A_{m+1}\right) \leqslant f\left(M_{1}\left(A_{m}\right)\right)$ and we are done.

This proposition solves the case $M_{1}(A)<1$. We still have to deal with the case $M_{1}(A)=1$, that is when all eigenvalues of $A$ are 1 . In this case, the inequality $\rho(A-I d) \geqslant 1-M_{1}(A)$ cannot help. Thus, we have to investigate in detail the decomposition of $A$ into Jordan blocks.

Let $J(\alpha, s) \in G L_{s}(\mathbb{C})$ be the Jordan block of size $s \times s$ and having eigenvalue $\alpha$, that is, the diagonal is composed only of values $\alpha$ and the entries directly above and to the right of the diagonal are 1 . We use the following recent description of the tensor product of Jordan blocks (surprisingly, the proof of this fact is quite involved). 
Theorem 5.7. ([MaV1, Theorem 2], [IiIwa09, Theorem 2.0.1]) For $s, t \in \mathbb{N}, s \leqslant t$ and $\alpha, \beta \in \mathbb{C}^{*}$ we have:

$$
J(\alpha, s) \otimes J(\beta, t)=\oplus_{i=1}^{s} J(\alpha \beta, s+t+1-2 i) .
$$

From now on, $A$ is a matrix in $G L_{n}(\mathbb{C})$ such that $M_{1}(A)=1$. Denote by $J(A)$ the number of Jordan blocks in $A$ divided by $n$. Then $\rho(A-I d)=1-J(A)$.

Proposition 5.8. If $M_{1}(A)=1$ then $J(A \otimes A) \leqslant J(A)$ and $J(A \otimes A) \leqslant J(A)^{2}+(1-$ $J(A))^{2}$.

Proof. For $i \in \mathbb{N}^{*}$ let $c_{i}$ be the number of Jordan blocks in $A$ of size $i$. Then $\sum_{i} i c_{i}=n$ and $\sum_{i} c_{i}=n J(A)$. Also $A=\oplus_{i} J(1, i) \otimes I d_{c_{i}}$. Then:

$$
A \otimes A=\oplus_{i, j} J(1, i) \otimes J(1, j) \otimes I d_{c_{i} c_{j}}
$$

According to the previous theorem the number of Jordan blocks in the matrix $J(1, i) \otimes$ $J(1, j)$ is $\min \{i, j\}$, so $n^{2} J(A \otimes A)=\sum_{i, j} c_{i} c_{j} \min \{i, j\}$. Then:

$$
n^{2} J(A \otimes A)=\sum_{i<j} c_{i} c_{j} i+\sum_{j<i} c_{i} c_{j} j+\sum_{i} c_{i}^{2} i=\sum_{i} i c_{i}^{2}+2 \sum_{i<j} i c_{i} c_{j} .
$$

Note that $n^{2}\left[J(A)^{2}+(1-J(A))^{2}\right]=(n J(A))^{2}+(n-n J(A))^{2}$, so:

$$
\begin{aligned}
n^{2}\left(J(A)^{2}+(1-J(A))^{2}\right) & =\left(\sum_{i} c_{i}\right)^{2}+\left(\sum_{i}(i-1) c_{i}\right)^{2}=\sum_{i} c_{i}^{2}+2 \sum_{i<j} c_{i} c_{j}+ \\
& +\sum_{i}(i-1)^{2} c_{i}^{2}+2 \sum_{i<j}(i-1)(j-1) c_{i} c_{j} \\
& =\sum_{i}\left[(i-1)^{2}+1\right] c_{i}^{2}+2 \sum_{i<j}[(i-1)(j-1)+1] c_{i} c_{j} .
\end{aligned}
$$

For the second inequality we only need to see that for any $i \in \mathbb{N}$ and $i<j$ we have $i \leqslant(i-1)^{2}+1 \leqslant(i-1)(j-1)+1$. The first inequality is easy because:

$$
n^{2} J(A)=\left(\sum_{i} i c_{i}\right)\left(\sum_{j} c_{j}\right)=\sum_{i, j} i c_{i} c_{j}
$$

Proposition 5.9. Let $c>1 / 2$ be a constant $c \in(J(A), 1)$. Then $J\left(A_{m}\right)<f^{m-1}(c)$. 
Proof. For $m=1, A_{m}=A$ and $f^{0}(c)=c$, hence the result follows by hypothesis. Suppose now that $J\left(A_{m}\right)<f^{m-1}(c)$. If $J\left(A_{m}\right) \leqslant 1 / 2$, then $J\left(A_{m+1}\right) \leqslant 1 / 2$ and we are done as $f^{m}(c)$ is always strictly greater than $1 / 2$.

Assume that $J\left(A_{m+1}\right)>1 / 2$. The function $f$ is strictly increasing, so $f\left(J\left(A_{m}\right)\right)<$ $f^{m}(c)$. By the previous proposition, $J\left(A_{m+1}\right) \leqslant f\left(J\left(A_{m}\right)\right)$ and we are done.

5.2. Equivalent definition. This section is devoted to the proof of the following theorem which will provide the strengthening of Proposition 4.4

Theorem 5.10. Let $G$ be a countable linear sofic group. Then there exists a morphism $\Psi: G \rightarrow \Pi_{k \rightarrow \omega} G L_{n_{k}}(\mathbb{C}) / d_{\omega}$ such that $d_{\omega}(\Psi(g), I d) \geqslant \frac{1}{4}$ for any $g \neq e$.

Proof. Let $\Theta: G \rightarrow \prod_{k \rightarrow \omega} G L_{n_{k}}(\mathbb{C}) / d_{\omega}$ be a linear sofic representation of $G$. Let $\theta^{k}(g) \in G L_{n_{k}}$ be such that $\Theta(g)=\Pi_{k \rightarrow \omega} \theta^{k}(g) / d_{\omega}$. Define $\theta_{1}^{k}(g):=\theta^{k}(g)$ and $\theta_{m+1}^{k}(g):=\theta_{m}^{k}(g) \otimes \theta_{m}^{k}(g)$. Notice that the matrix dimension of $\theta_{m}^{k}(g)$ is $n_{k}^{2^{m-1}}$.

Construct the linear sofic representation:

$$
\Theta_{m}: G \rightarrow \Pi_{k \rightarrow \omega} G L_{n_{k}^{2 m-1}}(\mathbb{C}) / d_{\omega}, \quad \Theta_{m}(g)=\Pi_{k \rightarrow \omega} \theta_{m}^{k}(g) / d_{\omega},
$$

and take the ultraproduct of these representations:

$$
\begin{gathered}
\Psi_{1}: G \rightarrow \prod_{(m, k) \rightarrow \omega \otimes \omega} G L_{n_{k}^{2 m-1}}(\mathbb{C}) / d_{\omega \otimes \omega}, \text { where } \\
\Psi_{1}(g)=\prod_{m \rightarrow \omega} \Theta_{m}(g) / d_{\omega}=\prod_{(m, k) \rightarrow \omega \otimes \omega} \theta_{m}^{k}(g) / d_{\omega \otimes \omega} .
\end{gathered}
$$

Also construct an amplification of $\Theta$ to this sequence of matrix dimensions:

$$
\begin{gathered}
\Psi_{2}: G \rightarrow \Pi_{(m, k) \rightarrow \omega \otimes \omega} G L_{n_{k}^{2 m-1}}(\mathbb{C}) / d_{\omega \otimes \omega}, \text { where } \\
\Psi_{2}(g)=\Pi_{(m, k) \rightarrow \omega \otimes \omega} \theta^{k}(g) \otimes I d_{n_{k}^{2^{m-1}-1}} / d_{\omega \otimes \omega} .
\end{gathered}
$$

Define $\Psi=\Psi_{1} \oplus \Psi_{2}, \Psi: G \rightarrow \Pi_{(m, k) \rightarrow \omega \otimes \omega} G L_{2 n_{k}^{2 m-1}}(\mathbb{C}) / d_{\omega \otimes \omega}$ such that:

$$
\rho_{\omega \otimes \omega}(\Psi(g)-I d)=\frac{1}{2}\left(\rho_{\omega \otimes \omega}\left(\Psi_{1}(g)-I d\right)+\rho_{\omega \otimes \omega}\left(\Psi_{2}(g)-I d\right)\right) .
$$

Claim 5.11. For any $g \in G$, we have $\rho_{\omega \otimes \omega}(\Psi(g)-I d) \geqslant 1 / 4$.

Assume that $\lim _{k \rightarrow \omega} M_{1}\left(\theta^{k}(g)\right) \leqslant 1 / 2$. Then $\lim _{n \rightarrow \omega} \lim _{k \rightarrow \omega} M_{1}\left(\theta^{k}(g) \otimes I d\right) \leqslant 1 / 2$. It follows that $\rho_{\omega \otimes \omega}\left(\Psi_{2}(g)-I d\right) \geqslant 1 / 2$, hence $\rho_{\omega \otimes \omega}(\Psi(g)-I d) \geqslant 1 / 4$ and we are done. We are left with the case $\lim _{k \rightarrow \omega} M_{1}\left(\theta^{k}(g)\right)>1 / 2$. 
Assume that $\lim _{k \rightarrow \omega} M_{1}\left(\theta^{k}(g)\right)<1$. Then there exist $c \in(1 / 2,1)$ and $F \in \omega$ such that $1 / 2<M_{1}\left(\theta^{k}(g)\right)<c$ for all $k \in F$. It follows by Lemma 5.6 that:

$$
M_{1}\left(\theta_{m}^{k}(g)\right)<f^{m-1}(c), \quad \forall k \in F, m \in \mathbb{N} .
$$

Then $\lim _{k \rightarrow \omega} M_{1}\left(\theta_{m}^{k}(g)\right) \leqslant f^{m-1}(c)$ for all $m \in \mathbb{N}$. We get the inequality:

$$
\lim _{m \rightarrow \omega} \lim _{k \rightarrow \omega} M_{1}\left(\theta_{m}^{k}(g)\right) \leqslant \lim _{m \rightarrow \omega} f^{m-1}(c)=1 / 2 .
$$

As a consequence $\rho_{\omega \otimes \omega}\left(\Psi_{1}(g)-I d\right) \geqslant 1 / 2$, so we have $\rho_{\omega \otimes \omega}(\Psi(g)-I d) \geqslant 1 / 4$.

We are left with the case $\lim _{k \rightarrow \omega} M_{1}\left(\theta^{k}(g)\right)=1$. We can assume that $M_{1}\left(\theta^{k}(g)\right)=1$ for each $k$. It follows that $M_{1}\left(\theta_{m}^{k}(g)\right)=1$ for each $m$ and $k$. The proof is similar to the previous case, using $J\left(\theta_{m}^{k}(g)\right)$ instead of $M_{1}\left(\theta_{m}^{k}(g)\right)$, the equation $\rho(A)=1-J(A)$, and Proposition 5.9 instead of Proposition 5.6.

The structure of the group does not play a role in the proof as $\Theta\left(g_{1}\right)$ does not interact with $\Theta\left(g_{2}\right)$ for $g_{1} \neq g_{2}$. The construction is possible even if we have just a subset of our group.

Proposition 5.12. Let $G$ be a countable group and let $E$ be a subset of $G$. Consider a function $\Phi: E \rightarrow \prod_{k \rightarrow \omega} G L_{n_{k}}(\mathbb{C}) / d_{\omega}$ such that $\Phi(g) \Phi(h)=\Phi(g h)$ whenever $g, h, g h \in$ $E$. Then there exists $\Psi: E \rightarrow \Pi_{k \rightarrow \omega} G L_{m_{k}}(\mathbb{C}) / d_{\omega}$ such that $\Psi(g) \Psi(h)=\Psi(g h)$ whenever $g, h, g h \in E$ and:

$$
\begin{aligned}
& \Phi(g)=\Phi(h) \Longrightarrow \Psi(g)=\Psi(h) \\
& \Phi(g) \neq \Phi(h) \Longrightarrow d_{\omega}(\Psi(g), \Psi(h)) \geqslant \frac{1}{4} .
\end{aligned}
$$

Now we can provide a stronger version of the algebraic characterization of linear soficity contained in Proposition 4.4 using this extra information that we obtained.

Proposition 5.13. A group $G$ is linear sofic if and only if for any finite subset $E \subset G$ and for any $\varepsilon>0$ there exists $n \in \mathbb{N}$ and a function $\phi: E \rightarrow G L_{n}(\mathbb{C})$ such that:

(1) $\forall g, h, g h \in E$ we have $\rho(\phi(g) \phi(h)-\phi(g h))<\varepsilon$;

(2) $\forall g \in E$ we have $\rho(1-\phi(g))>\frac{1}{4}-\varepsilon$. 


\section{RATIONAL LINEAR SOFICITY}

This section is devoted to proving that in the definition of linear sofic group (see Definition 4.1) we can use the groups $G L_{n}(\mathbb{Q})$ endowed with the rank metric. In other words, the existence of a complex linear sofic representation is equivalent to the existence of a rational linear sofic representation.

Lemma 6.1. A group $G$ is linear sofic if and only iffor any finite subset $E \subset G$ and for any $\varepsilon>0$ there exist $n \in \mathbb{N}$ and a function $\phi: E \rightarrow G L_{n}(\mathbb{R})$ such that:

(1) $\forall g, h, g h \in E$ at least $(1-\varepsilon) n$ columns of the matrix $\phi(g) \phi(h)$ are equal to the corresponding columns in $\phi(g h)$;

(2) $\forall g \in E$ we have $\rho(1-\phi(g))>\frac{1}{4}-\varepsilon$.

Proof. Elements in $G L_{n}(\mathbb{R})$ are invertible linear transformations on $\mathbb{R}^{n}$. These elements are matrices as soon as we fix a basis for the vector space $\mathbb{R}^{n}$. As the second condition does not depend on the particular choice of a basis, we only need to concentrate our efforts to constructing a basis such that the first condition holds.

Let $E_{1}=\left\{(g, h) \in E^{2} \mid g h \in E\right\}$. Then $E_{1}$ is a finite set. Take $\delta:=\varepsilon /\left|E_{1}\right|$. Apply Proposition 5.13 for $E$ and $\delta$ to get $n \in \mathbb{N}$ and a function $\phi: E \rightarrow G L_{n}(\mathbb{R})$ (use Observation 4.3 to replace $\mathbb{C}$ by $\mathbb{R}$ ). For each $(g, h) \in E_{1}$ let $V_{g, h} \subset \mathbb{R}^{n}$ be the linear subspace on which $\phi(g) \phi(h)=\phi(g h)$. By condition (1) of Proposition 5.13 it follows that $\operatorname{dim} V_{g, h}>(1-\delta) n$ for any $(g, h) \in E_{1}$. Let $V=\bigcap_{(g, h) \in E_{1}} V_{g, h}$. Then $\operatorname{dim} V>\left(1-\left|E_{1}\right| \delta\right) n=(1-\varepsilon) n$. Choose a basis in $V$ and complete it to a basis in $\mathbb{R}^{n}$.

Using this basis we can see elements in $\phi(E)$ as matrices. It is clear now by construction that the first condition holds.

We denote by $\overline{\mathbb{Q}}$ the field of real algebraic numbers. The next step in the proof is to replace the function $\phi: E \rightarrow G L_{n}(\mathbb{R})$ by another function $\psi: E \rightarrow G L_{n}(\overline{\mathbb{Q}})$. In order to achieve this we will use the following variant of the fundamental result of real semialgebraic geometry, so-called Positivstellensatz.

Theorem 6.2. (["BCR98, Theorem 4.4.2, p. 92]) Let $R$ be a real closed field. Let $\left(f_{j}\right)_{j=1, \ldots, s},\left(g_{k}\right)_{k=1, \ldots, t}$, and $\left(h_{l}\right)_{l=1, \ldots, u}$ be finite families of polynomials in $R\left[X_{1}, \ldots, X_{d}\right]$. Denote by $P$ the cone generated by $\left(f_{j}\right)_{j=1, \ldots, s}$, by $M$ the multiplicative monoid generated by $\left(g_{k}\right)_{k=1, \ldots, t}$, and by $I$ the ideal generated by $\left(h_{l}\right)_{l=1, \ldots, u}$. Then the following properties are equivalent: 
(1) The set $\left\{x \in R^{d} \mid f_{j}(x) \geqslant 0 \forall j, g_{k}(x) \neq 0 \forall k, h_{l}(x)=0 \forall l\right\}$ is empty.

(2) There exist $f \in P, g \in M$, and $h \in I$ such that $f+g^{2}+h=0$.

Corollary 6.3. Let $\left(g_{k}\right)_{k=1, \ldots, t}$ and $\left(h_{l}\right)_{l=1, \ldots, u}$ be finite families of polynomials in $\mathbb{Q}\left[X_{1}, \ldots, X_{d}\right]$. If there exists a real solution $x \in \mathbb{R}^{d}$ to the system:

$$
\begin{array}{ll}
g_{k}(x) \neq 0 & k=1, \ldots, t, \\
h_{l}(x)=0 & l=1, \ldots, u,
\end{array}
$$

then there is also a solution $x \in \overline{\mathbb{Q}}^{d}$.

Proof. Let $P$ be the smallest cone in $\overline{\mathbb{Q}}\left[X_{1}, \ldots, X_{d}\right]$, that is $P$ contains squares in $\overline{\mathbb{Q}}\left[X_{1}, \ldots, X_{d}\right]$ and it is closed under addition and multiplication by positive scalars. Let also $M$ be the multiplicative monoid generated by $\left(g_{k}\right)_{k=1, \ldots, t}$ and $I$ the ideal generated by $\left(h_{l}\right)_{l=1, \ldots, u}$ in $\overline{\mathbb{Q}}\left[X_{1}, \ldots, X_{d}\right]$.

If there is no solution $x \in \overline{\mathbb{Q}}^{d}$ to the system above, then according to the previous theorem there exist $f \in P, g \in M$, and $h \in I$ such that $f+g^{2}+h=0$. However, this equation also holds in $\mathbb{R}\left[X_{1}, \ldots, X_{d}\right]$ so there should not exist a solution $x \in \mathbb{R}^{d}$.

Proposition 6.4. A group $G$ is linear sofic if and only if for any finite subset $E \subset G$ and for any $\varepsilon>0$ there exist $n \in \mathbb{N}$ and a function $\phi: E \rightarrow G L_{n}(\overline{\mathbb{Q}})$ such that:

(1) $\forall g, h, g h \in E$ we have $\rho(\phi(g) \phi(h)-\phi(g h))<\varepsilon$;

(2) $\forall g \in E$ we have $\rho(1-\phi(g))>\frac{1}{4}-\varepsilon$.

Proof. Using $E$ and $\varepsilon$, apply Lemma 6.1 to get a function $\phi: E \rightarrow G L_{n}(\mathbb{R})$. Recall from the proof of that lemma that $E_{1}=\left\{(g, h) \in E^{2} \mid g h \in E\right\}$.

We regard conditions (1) and (2) of Lemma 6.1 as a system of equations and nonequations. The variables of this system are the $n^{2}|E|$ entries of matrices in $\phi(E)$.

Condition $(1)$ in Lemma 6.1 provides more than $(1-\varepsilon) n$ equations for each pair $(g, h) \in E_{1}$. These equations are enough to deduce that $\rho(\phi(g) \phi(h)-\phi(g h))<\varepsilon$. For each $g \in E$ choose a minorant of $1-\phi(g)$ of size greater than $(1 / 4-\varepsilon) n \times(1 / 4-\varepsilon) n$ of nonzero determinant. This information will provide a non-equation.

Apply now the previous corollary to get a solution to our system in $\overline{\mathbb{Q}}^{n^{2}|E|}$. Using this solution we construct a map $\phi: E \rightarrow G L_{n}(\overline{\mathbb{Q}})$ with the required properties. 
Theorem 6.5. Let $G$ be a linear sofic group. Then there exists an injective morphism $\Theta: G \rightarrow \prod_{k \rightarrow \omega} G L_{n_{k}}(\mathbb{Q})$.

Proof. Fix a finite subset $E \subset G$ and $\varepsilon>0$. By the previous proposition, we obtain a $\operatorname{map} \phi: E \rightarrow G L_{n}(\overline{\mathbb{Q}})$, satisfying the algebraic definition of linear soficity. We replace $\overline{\mathbb{Q}}$ by $F$, the field generated by the $n^{2}|E|$ entries of the matrices in $\phi(E)$. Being a finitely generated algebraic extension over $\mathbb{Q}$, the field $F$ is also a vector space over $\mathbb{Q}$ of finite dimension. Then, we proceed as in Observation 4.3 and we get a required function $\psi: E \rightarrow G L_{n}(\mathbb{Q})$ having the same properties as in the algebraic definition.

\section{LINEAR SOFIC GROUPS AND ALGEBRAS}

This section is devoted to proving Theorem 1.2 that a group $G$ is linear sofic if and only if $\mathbb{C} G$ is a linear sofic algebra. While the "if" part follows directly from Proposition 2.6, the "only if" part is much more involved.

Notation 7.1. If $\Theta: G \rightarrow \prod_{k \rightarrow \omega} G L_{n_{k}}(F) / d_{\omega}$ is a group morphism we denote by $\widetilde{\Theta}$ its extension to the group algebra:

$$
\widetilde{\Theta}: F(G) \rightarrow \Pi_{k \rightarrow \omega} M_{n_{k}}(F) / \operatorname{Ker} \rho_{\omega}, \widetilde{\Theta}\left(\sum a_{i} u_{g_{i}}\right):=\sum a_{i} \Theta\left(g_{i}\right),
$$

where $a_{i} \in F, g_{i} \in G$ and $u_{g_{i}}$ is the element in the group algebra corresponding to $g_{i}$.

Example 7.2. If $\Theta$ is injective on $G$ it does not follow that $\widetilde{\Theta}$ is injective on $F(G)$. As an easy example consider $\Theta: \mathbb{Z} \rightarrow \prod_{k \rightarrow \omega} G L_{k}(\mathbb{R}) / d_{\omega}, \Theta(i)=2^{i} I d$, for $i \in \mathbb{Z}$. Then for $u_{1}-2 u_{0} \in \mathbb{R}(\mathbb{Z})$ we have $\widetilde{\Theta}\left(u_{1}-2 u_{0}\right)=2 I d-2 I d=0$.

The proof relies on the direct sum and tensor product of elements in ultraproduct of matrices. Here are variants of (4) and (5) of Proposition 2.2 extended to ultraproducts.

Proposition 7.3. Let $u=\left(u_{k}\right)_{k} \in \Pi_{k \rightarrow \omega} M_{n_{k}}(F) / \operatorname{Ker} \rho_{\omega}$ and $v=\left(v_{k}\right)_{k} \in$ $\Pi_{k \rightarrow \omega} M_{m_{k}}(F) / K \operatorname{er} \rho_{\omega}$. Then:

$$
\begin{array}{ll}
u \oplus v=\left(u_{k} \oplus v_{k}\right)_{k} \in \Pi_{k \rightarrow \omega} M_{n_{k}+m_{k}}(F) / \operatorname{Ker} \rho_{\omega} & ; \rho_{\omega}(u \oplus v)=\frac{n_{k} \rho_{\omega}(u)+m_{k} \rho_{\omega}(v)}{n_{k}+m_{k}} ; \\
u \otimes v=\left(u_{k} \otimes v_{k}\right)_{k} \in \Pi_{k \rightarrow \omega} M_{n_{k} m_{k}}(F) / K \operatorname{Ker} \rho_{\omega} \quad ; \rho_{\omega}(u \otimes v)=\rho_{\omega}(u) \cdot \rho_{\omega}(v) .
\end{array}
$$

Theorem 7.4. Let $\Theta: G \rightarrow \prod_{k \rightarrow \omega} G L_{n_{k}}(F) / d_{\omega}$ be an injective group morphism. Then there exists an injective algebra morphism $\Psi: F(G) \rightarrow \Pi_{k \rightarrow \omega} M_{m_{k}}(F) / \operatorname{Ker} \rho_{\omega}$. 
Proof. Let $\theta_{k}: G \rightarrow G L_{n_{k}}(F)$ be some functions such that $\Theta=\Pi_{k \rightarrow \omega} \theta_{k} / d_{\omega}$. Then $\Theta \otimes \Theta: G \rightarrow \prod_{k \rightarrow \omega} G L_{n_{k}^{2}}(F) / d_{\omega}$, defined by $\Theta \otimes \Theta(g)=\Pi \theta_{k}(g) \otimes \theta_{k}(g) / d_{\omega}$ is a linear sofic representation of $G$. For every $i \in \mathbb{N}$ define a map

$$
\theta_{k}^{i}: G \rightarrow G L_{n_{k}^{i}}(F), \quad \theta_{k}^{i}(g):=\theta_{k}(g) \otimes \ldots \otimes \theta_{k}(g) \text { (i times tensor product), }
$$

and set $\Theta^{i}=\prod_{k \rightarrow \omega} \theta_{k}^{i} / d_{\omega}$. For $m \geqslant i$ define

$$
\theta_{k}^{i, m}: G \rightarrow G L_{n_{k}^{m}}(F), \quad \theta_{k}^{i, m}(g):=\theta_{k}^{i}(g) \otimes I d_{n_{k}^{m-i}} .
$$

The meaning of this definition is to bring the first $m \theta_{k}^{i}$, s into the same matrix dimension.

Now define $\phi_{k}: G \rightarrow G L_{n_{k}^{k} 2^{k}}$ by:

$$
\phi_{k}=\left(\theta_{k}^{1, k} \otimes I d_{2^{k-1}}\right) \oplus\left(\theta_{k}^{2, k} \otimes I d_{2^{k-2}}\right) \oplus \ldots \oplus\left(\theta_{k}^{k, k} \otimes I d_{2^{0}}\right) \oplus\left(I d_{n_{k}^{k}} \otimes I d_{2^{0}}\right)
$$

and set $\Phi=\prod_{k \rightarrow \omega} \phi_{k} / d_{\omega}$.

The reason for this definition is the relation:

$$
\rho_{\omega}(\widetilde{\Phi}(f))=\sum_{i=1}^{\infty} \frac{1}{2^{i}} \rho_{\omega}\left(\widetilde{\Theta}^{i}(f)\right),
$$

for any $f \in F(G)$. Before proving this equality let us state our crucial claim.

Claim 7.5. $\widetilde{\Phi}: F(G) \rightarrow \prod_{k \rightarrow \omega} M_{n_{k}^{k} 2^{k}}(F) / \operatorname{Ker} \rho_{\omega}$ is injective.

We now prove the stated relation:

$$
\begin{aligned}
\rho_{\omega}(\widetilde{\Phi}(f)) & =\lim _{k \rightarrow \omega} \frac{r k\left(\tilde{\phi}_{k}(f)\right)}{n_{k}^{k} 2^{k}}=\lim _{k \rightarrow \omega} \frac{1}{n_{k}^{k} 2^{k}} \sum_{i=1}^{k} r k\left(\tilde{\theta}_{k}^{i, k} \otimes I_{2^{k-i}}(f)\right) \\
& =\lim _{k \rightarrow \omega} \sum_{i=1}^{k} \frac{1}{2^{i} n_{k}^{k}} r k\left(\tilde{\theta}_{k}^{i, k}(f)\right)=\lim _{k \rightarrow \omega} \sum_{i=1}^{k} \frac{1}{2^{i} n_{k}^{i}} r k\left(\tilde{\theta}_{k}^{i}(f)\right) \\
& =\sum_{i=1}^{\infty} \frac{1}{2^{i}} \rho_{\omega}\left(\widetilde{\Theta}^{i}(f)\right)
\end{aligned}
$$

Assume now that $f \in F(G)$ such that $\rho_{\omega}(\widetilde{\Phi}(f))=0$. Then $\rho_{\omega}\left(\widetilde{\Theta}^{i}(f)\right)=0$ for any $i$. In order to present our injectivity argument in a transparent way we shall assume that $f=a_{1} u_{1}+a_{2} u_{2}+a_{3} u_{3}$, where $a_{i}$ are nonzero elements of $F$ and $u_{i}$ are invertible elements in the group algebra corresponding to distinct elements in the group $G$. We know that:

$$
\begin{aligned}
& a_{1} \Theta\left(u_{1}\right)+a_{2} \Theta\left(u_{2}\right)+a_{3} \Theta\left(u_{3}\right)=0 \\
& a_{1} \Theta\left(u_{1}\right) \otimes \Theta\left(u_{1}\right)+a_{2} \Theta\left(u_{2}\right) \otimes \Theta\left(u_{2}\right)+a_{3} \Theta\left(u_{3}\right) \otimes \Theta\left(u_{3}\right)=0
\end{aligned}
$$


(3)

$a_{1} \Theta\left(u_{1}\right) \otimes \Theta\left(u_{1}\right) \otimes \Theta\left(u_{1}\right)+a_{2} \Theta\left(u_{2}\right) \otimes \Theta\left(u_{2}\right) \otimes \Theta\left(u_{2}\right)+a_{3} \Theta\left(u_{3}\right) \otimes \Theta\left(u_{2}\right) \otimes \Theta\left(u_{3}\right)=0$

Amplifying the first equation by $\Theta\left(u_{3}\right)$ and subtracting it from the second we get:

$$
a_{1} \Theta\left(u_{1}\right) \otimes\left(\Theta\left(u_{1}\right)-\Theta\left(u_{3}\right)\right)+a_{2} \Theta\left(u_{2}\right) \otimes\left(\Theta\left(u_{2}\right)-\Theta\left(u_{3}\right)\right)=0
$$

Applying the same operation to equations (2) and (3), we get:

$$
a_{1} \Theta\left(u_{1}\right) \otimes \Theta\left(u_{1}\right) \otimes\left(\Theta\left(u_{1}\right)-\Theta\left(u_{3}\right)\right)+a_{2} \Theta\left(u_{2}\right) \otimes \Theta\left(u_{2}\right) \otimes\left(\Theta\left(u_{2}\right)-\Theta\left(u_{3}\right)\right)=0
$$

Now we amplify equation (4) with $\Theta\left(u_{2}\right)$ between the already existing tensor product, and subtract it from equation (5) to get:

$$
a_{1} \Theta\left(u_{1}\right) \otimes\left(\Theta\left(u_{1}\right)-\Theta\left(u_{2}\right)\right) \otimes\left(\Theta\left(u_{1}\right)-\Theta\left(u_{3}\right)\right)=0
$$

As $a_{1} \neq 0$ and $\Theta\left(u_{1}\right)$ is invertible, we get that $\Theta\left(u_{1}\right)=\Theta\left(u_{2}\right)$ or $\Theta\left(u_{1}\right)=\Theta\left(u_{3}\right)$. This contradicts the injectivity of $\Theta$. This procedure applies to any $f$ with an arbitrary large (finite) support.

The key of the proof is the construction of the representation $\widetilde{\Phi}$ out of a sequence of maps $\widetilde{\Theta}^{i}$ such that $\widetilde{\Phi}(x)=0$ if and only if $\widetilde{\Theta}^{i}(x)=0$ for all $i$. This is a construction that can be performed in general and we record it here for a later use.

Proposition 7.6. Let $\left\{\Theta^{i}\right\}_{i}, \Theta^{i}: A \rightarrow \Pi_{k \rightarrow \omega} M_{n_{i, k}} /$ Ker $\rho_{\omega}$ be a sequence of morphisms of an algebra $A$. Then there exists a morphism $\Phi$ of $A$ such that $\rho_{\omega}(\Phi(x))=$ $\sum_{i=1}^{\infty} \frac{1}{2^{i}} \rho_{\omega}\left(\Theta^{i}(x)\right)$ for any $x \in A$. In particular, $\Phi(x)=0$ if and only if $\Theta^{i}(x)=0$ for all $i$. Moreover, if $\left\{\Theta^{i}\right\}_{i}$ are unital morphisms, then $\Phi$ can be taken unital.

Corollary 7.7. A group $G$ is linear sofic if and only if $\mathbb{C} G$ is a linear sofic algebra.

Proof. The direct implication is the previous theorem for $F=\mathbb{C}$. The reverse implication immediately follows from Proposition 2.6 .

Our previous theorem also provides a new proof of the result of Elek and Szabo [ElSz04].

Corollary 7.8. Sofic groups satisfy Kaplansky's direct finiteness conjecture. 
Proof. Let $F$ be a field and $G$ be a sofic group. Same arguments as in Proposition 4.5 show that there exists an injective group morphism $\Theta: G \rightarrow$ $\prod_{k \rightarrow \omega} G L_{n_{k}}(F) / d_{\omega}$. The previous theorem provides an injective algebra homomorphism $\Psi: F(G) \rightarrow \Pi_{k \rightarrow \omega} M_{m_{k}}(F) / \operatorname{Ker} \rho_{\omega}$. However, $\Pi_{k \rightarrow \omega} M_{m_{k}}(F) / \operatorname{Ker} \rho_{\omega}$ is stably finite by Proposition 2.8. Thus, $F(G)$ is actually stably finite in this case.

Question 7.9. Do linear sofic groups satisfy Kaplansky's direct finiteness conjecture?

See also comments following Question 8.6 below.

\section{LINEAR SOFIC IMPLIES WEAKLY SOFIC}

Here we prove that a linear sofic group is weakly sofic. The proof is an adaptation of the proof of Malcev's theorem 2 presented in [PeKw09. Theorem 1.4]. Let us recall the definition of weakly sofic group.

Definition 8.1. (c.f. [GIRi08, Definition 4.1]) A group $G$ is weakly sofic if it can be embedded in a metric ultraproduct of finite groups, each equipped with a bi-invarant metric.

The original definition in [GIRi08] is algebraic and uses a constant length function (as discussed before Proposition 4.4). It is equivalent to its ultraproduct version above by standard amplification argument [Pe0]. Indeed, the direct product of finite groups is obviously finite and one can define a bi-invariant distance on the direct product as the sum of the bi-invariant metrics on the factors.

Theorem 8.2. If $G$ is a linear sofic group then there exists $\left(F_{k}\right)_{k}$ a sequence of finite fields and an injective group morphism $\Phi: G \rightarrow \prod_{k \rightarrow \omega} G L_{n_{k}}\left(F_{k}\right) / d_{\omega}$.

Proof. Let $\theta_{k}: G \rightarrow G L_{n_{k}}(\mathbb{C})$ be some functions such that $\Theta=\Pi_{k \rightarrow \omega} \theta_{k} / d_{\omega}$ is an injective homomorphism given by linear soficity of $G$. Let $G=\bigcup_{k} B_{k}$, where $\left(B_{k}\right)_{k}$ is an increasing sequence of finite subsets of $G$ such that $B_{k}^{-1}=B_{k}$ and $e \in B_{k}$. Let $R_{k} \subset \mathbb{C}$ be the ring generated by all the entries of $\theta_{k}(s)$ with $s \in B_{k}$. Because it is finitely generated, $R_{k}$ is a Jacobson ring. We can view $\theta_{k}$ as a map from $B_{k}$ to $G L_{n_{k}}\left(R_{k}\right)$.

\footnotetext{
${ }^{2}$ Malcev proves that every finitely generated subgroup of the linear group $G L_{n}(F)$ is residually finite.
} 
Claim 8.3. There exists $m_{k} \subset R_{k}$ a maximal ideal such that if we reduce $\theta_{k}$ modulo $m_{k}$ to get the induced map $\phi_{k}: B_{k} \rightarrow G L_{n_{k}}\left(R_{k} / m_{k}\right)$ we get:

$$
r k\left(I-\theta_{k}(s)\right)=r k\left(I-\phi_{k}(s)\right) \forall s \in B_{k} .
$$

For $s \in B_{k}$ let $a_{s}=r k\left(I-\theta_{k}(s)\right)$ and choose $A_{s}$ an $a_{s} \times a_{s}$ submatrix of $\theta_{k}(s)$ such that $b_{s}=\operatorname{det} A_{s} \neq 0$. Let $c=\Pi_{s \in B_{k}} b_{s}$ and choose $m_{k} \subset R_{k}$ a maximal ideal such that $c \notin m_{k}$. Then $b_{s} \notin m_{k}$ for any $s \in B_{k}$ so indeed $r k\left(I-\theta_{k}(s)\right)=r k\left(I-\phi_{k}(s)\right)$.

Since $m_{k}$ is a maximal ideal, $R_{k} / m_{k}$ is a field. It is a well-known non-trivial fact that a finitely generated ring, that is also a field, is finite. It follows that $R_{k} / m_{k}$ is finite.

Define $\Phi=\prod_{k \rightarrow \omega} \phi_{k} / d_{\omega}$ and note that in general if $s, t, s t \in B_{k}$ then $r k\left(\phi_{k}(s t)-\right.$ $\left.\phi_{k}(s) \phi_{k}(t)\right) \leqslant r k\left(\theta_{k}(s t)-\theta_{k}(s) \theta_{k}(t)\right)$. This implies that $\Phi$ is still a homomorphism and the claim shows that $\Phi$ is injective.

Observation 8.4. Every finite field $F$ is a finite dimensional vector space over $\mathbb{Z} / p \mathbb{Z}$, where $p$ is the characteristic of $F$. Therefore, as in Observation 4.3, if we have an embedding $\phi: G \rightarrow \prod_{k \rightarrow \omega} G L_{n_{k}}\left(F_{k}\right) / d_{\omega}$ with $F_{k}$ finite fields, then we can construct $\psi: G \rightarrow \prod_{k \rightarrow \omega} G L_{m_{k}}\left(\mathbb{Z} / p_{k} \mathbb{Z}\right) / d_{\omega}$, where $\left(p_{k}\right)_{k}$ is a sequence of prime numbers.

Question 8.5. Are all linear sofic groups indeed sofic?

For this question the tensor product is not a useful tool. Suppose that we have a map $\theta: E \rightarrow G L_{n}(\mathbb{C})$ from a finite subset $E$ of a linear sofic group $G$. We want to construct a new map from $E$ into $S_{n}$. As permutation matrices are diagonalizable, we can first try to construct a map using only diagonalizable matrices.

If $A \in M_{n}(\mathbb{C})$ let $A=U T U^{-1}$ be the canonical Jordan decomposition of $A$. Let $\operatorname{Diag}(T)$ be the diagonal matrix obtained by taking only the entries on the diagonal of $T$. Then $\rho\left(A-U \operatorname{Diag}(T) U^{-1}\right)=1-J(A)$, where $J(A)$ is the number of Jordan blocks in $A$ divided by $n$ as defined in Section 5. In the rank metric, this is the lower bound for $\rho(A-D)$, where $D$ is any diagonalizable matrix. This follows from Theorem 2 of [G1Ri09] or it can be checked directly. In Section 5, we proved that $J(A \otimes A) \leqslant J(A)$. Therefore, taking the tensor product will only increase the rank distance from $A$ to a diagonalizable matrix, not reduce it.

Question 8.6. Let $G$ be a linear sofic group and $F$ a finite field. Does there exist an injective group morphism $\Phi: G \rightarrow \prod_{k \rightarrow \omega} G L_{n_{k}}(F) / d_{\omega}$ ? 
Sofic groups have this property and this is the only property that we used in our proof of Kaplansky's direct finiteness conjecture, Corollary 7.8, A positive answer to this question will immediately imply that linear sofic groups do satisfy Kaplansky's direct finiteness conjecture. That would give a positive answer to Question 7.9 .

\section{PermanenCE PROPERTIES}

Here we shall prove various permanence properties for linear sofic groups and algebras. Due to Theorem 1.2 many permanence properties for linear sofic algebras can be transported to linear sofic groups.

Theorem 9.1. Subalgebras, direct product, inverse limits of linear sofic algebras are linear sofic. Same permanence properties hold also for linear sofic groups.

Proof. It is not hard to see that a subalgebra of a linear sofic algebra is linear sofic. Let $\left(A_{i}\right)_{i}$ be a sequence of linear sofic algebras and let $A=\Pi_{i} A_{i}$ be its direct product. Denote by $P_{j}: \Pi_{i} A_{i} \rightarrow A_{j}$ the projection to the $j$-th component. Let $\Theta_{i}: A_{i} \rightarrow \Pi_{k \rightarrow \omega} M_{n_{i, k}} / \operatorname{Ker} \rho_{\omega}$ be a linear sofic representation of $A_{i}$. Then $\left(\Theta_{i} \circ P_{i}\right)_{i}$ is a sequence of morphisms of the algebra $A$. Using Proposition 7.6 we construct $\Psi: A \rightarrow \Pi_{k \rightarrow \omega} M_{n_{k}} / \operatorname{Ker} \rho_{\omega}$ such that $\operatorname{Ker} \Psi=\bigcap_{i} \operatorname{Ker}\left(\Theta_{i} \circ P_{i}\right)=0$. It follows that $A$ is linear sofic. An inverse limit is a specific subalgebra of the direct product.

The second part of the theorem follows immediately from Theorem 1.2 and properties of group algebras for these constructions.

Theorem 9.2. Direct limit of linear sofic groups is again sofic.

Proof. For the proof we use Proposition 5.13, Note that Proposition 4.4 is not sufficient for this result.

Let $\left\{G_{i}\right\}_{i}$ be a family of linear sofic groups together with morphisms required to construct the group $G$, the direct limit of this family. Let $\psi_{i}: G_{i} \rightarrow G$ be the morphisms provided by the definition of the direct limit.

Let $E$ be a finite subset of $G$ and $\varepsilon>0$. There exists $i_{0} \in \mathbb{N}$ and $E_{0} \subset G_{i_{0}}$ such that $\psi_{i_{0}}: E_{0} \rightarrow E$ is a bijection. Apply Proposition 5.13 for $G_{i_{0}}, E_{0}$ and $\varepsilon$ to get $\phi: E_{0} \rightarrow G L_{n}(\mathbb{C})$. Then $\phi \circ \psi_{i_{0}}^{-1}: E \rightarrow G L_{n}(\mathbb{C})$ is the required function for $G, E$ and $\varepsilon$. 
Elek and Szabo proved that amenable extensions of sofic groups is again sofic [ElSz06]. The same result is true for linear sofic groups and our proof is a careful adaptation of the proof of the sofic case presented in [Oz09].

Theorem 9.3. Let $G$ be a countable group and $H$ a normal subgroup of $G$. If $H$ is linear sofic and $G / H$ is amenable then $G$ is linear sofic.

Proof. Let $\sigma: G / H \rightarrow G$ be a lift and define $\alpha: G \times G / H \rightarrow H$ by $\alpha(g, \gamma)=$ $\sigma\left(g \gamma^{-1}\right) g \sigma(\gamma)$. Then $\alpha$ satisfies the cocycle identity, $\alpha\left(g_{1} g_{2}, \gamma\right)=\alpha\left(g_{1}, g_{2} \gamma\right) \alpha\left(g_{2}, \gamma\right)$.

Let $E \subset G$ be a finite subset and $\varepsilon>0$. Let $F \subset G / H$ be such that $|g F \cap F|>$ $(1-\varepsilon)|F|$ for all $g \in E$. Then $\alpha(E, F) \subset H$ is finite. Use Proposition 5.13 and the linear soficity of $H$ to get $\phi: \alpha(E, F) \rightarrow G L_{n}(\mathbb{C})$. Construct $\psi: E \rightarrow M_{n \cdot|F|}(\mathbb{C})$ by:

$$
\psi(g)=\sum_{\gamma \in F \cap g^{-1} F} \phi(\alpha(g, \gamma)) \otimes e_{g \gamma, \gamma} .
$$

Here $e_{g \gamma, \gamma} \in M_{|F|}(\mathbb{C})$ is a unit matrix, that is a matrix having only one entry of 1 on the position $(g \gamma, \gamma)$. It is easy to compute $\rho(\psi(g))=\left|F \cap g^{-1} F\right| /|F|>1-\varepsilon$, so $\psi(g)$ is almost an element of $G L_{n|F|}$.

We want to show that $\psi\left(g_{1}\right) \psi\left(g_{2}\right)$ is close to $\psi\left(g_{1} g_{2}\right)$. By construction:

$$
\psi\left(g_{1}\right) \psi\left(g_{2}\right)=\sum_{\gamma_{1} \in F \cap g_{1}^{-1} F} \sum_{\gamma_{2} \in F \cap g_{2}^{-1} F} \phi\left(\alpha\left(g_{1}, \gamma_{1}\right)\right) \phi\left(\alpha\left(g_{2}, \gamma_{2}\right)\right) \otimes e_{g_{1} \gamma_{1}, \gamma_{1}} e_{g_{2} \gamma_{2}, \gamma_{2}} .
$$

Inside the sum we must have $\gamma_{1}=g_{2} \gamma_{2}$ in order to get a non trivial term.

$$
\psi\left(g_{1}\right) \psi\left(g_{2}\right)=\sum_{\gamma_{2} \in F \cap g_{2}^{-1} F \cap\left(g_{1} g_{2}\right)^{-1} F} \phi\left(\alpha\left(g_{1}, g_{2} \gamma_{2}\right)\right) \phi\left(\alpha\left(g_{2}, \gamma_{2}\right)\right) \otimes e_{g_{1} g_{2} \gamma_{2}, \gamma_{2}} .
$$

Also $\psi\left(g_{1} g_{2}\right)=\sum_{\gamma \in F \cap\left(g_{1} g_{2}\right)^{-1} F} \phi\left(\alpha\left(g_{1}, g_{2} \gamma\right) \alpha\left(g_{2}, \gamma\right)\right) \otimes e_{g_{1} g_{2} \gamma, \gamma}$. Comparing the two equations we get:

$$
\rho\left(\psi\left(g_{1}\right) \psi\left(g_{2}\right)-\psi\left(g_{1} g_{2}\right)\right) \leqslant \frac{1}{n|F|}(|F| n \varepsilon+\varepsilon|F|)<2 \varepsilon .
$$

We only need to show that $\rho(I d-\psi(g))$ is larger than a constant. If $g \in H$ then:

$\rho(I d-\psi(g))=\frac{1}{|F|} \rho\left(I d-\sum_{\gamma \in F} \phi(\alpha(g, \gamma)) \otimes e_{\gamma, \gamma}\right)=\frac{1}{|F|} \sum_{\gamma \in F} \rho\left(I d-\phi(\alpha(g, \gamma)) \geqslant \frac{1}{4}-\varepsilon\right.$.

Consider now the case $g \notin H$ such that $g \gamma \neq \gamma$ for any $\gamma \in F$. Let $x=\left(x_{\gamma}\right)_{\gamma} \in$ $\mathbb{C}^{n \cdot|F|}$ be a vector in $\operatorname{Ker}(I d-\psi(g))$. An easy computation will provide the equation $\phi(\alpha(g, \gamma))\left(x_{\gamma}\right)=x_{g \gamma}$ for $\gamma \in F \cap g^{-1} F$. This means that if we fix $x_{\gamma}$ then $x_{g \gamma}$ 
is completely determined. It follows that $\operatorname{dim} \operatorname{Ker}(\operatorname{Id}-\psi(g))$ can not be greater than $1 / 2$ minus some $\varepsilon$ due to the restriction $\gamma \in F \cap g^{-1} F$. So $\rho(I d-\psi(g))=$ $1-\operatorname{dim} \operatorname{Ker}(I d-\psi(g)) \geqslant 1 / 2-\varepsilon$.

\section{THE NUMBER OF UNIVERSAL LINEAR SOFIC GROUPS}

A universal linear sofic group is a metric ultraproduct of $\left(G L_{n_{k}}(F)\right)_{k}$ as defined in Definition 2.5. In [Lu11] Lupini proved that, under the failure of the Continuum Hypothesis $(\mathrm{CH})$, there are $2^{\aleph_{c}}$ metric ultraproducts of matrix algebras endowed with the metric induced by the rank (Definition 2.3), up to algebraic isomorphism. This result is based on methods of continuous logic developed in [FaSh09]. Here we extend Lupini's arguments to show that, assuming $\neg C H$, there are $2^{\aleph_{c}}$ universal linear sofic groups. Such results are not known for general weakly sofic groups when the approximating family of finite groups endowed with bi-invariant metrics is given.

Recall that by definition $\aleph_{c}:=2^{\aleph_{0}}$, where $\aleph_{0}$ is the cardinality of $\mathbb{N}$. If $a, b$ are elements of a group then $[a, b]$ is defined as $a b a^{-1} b^{-1}$.

In this section, $\left(n_{k}\right)_{k} \subset \mathbb{N}$ is a fixed strictly increasing sequence. We obtain nonisomorphic universal linear sofic groups by using different ultrafilters.

Proposition 10.1. ([Lu11, Corollary 2]) Let $\left(G_{n}\right)_{n}$ be a sequence of groups, each equipped with a bi-invarant metric, with uniformly bounded diameter. Suppose that for some constant $\gamma>0$ and every $l \in \mathbb{N}$, for all but finitely many $n \in \mathbb{N}, G_{n}$ contains sequences $\left(g_{n, i}\right)_{i=1}^{l}$ and $\left(h_{n, i}\right)_{i=1}^{l}$ such that, for every $1 \leqslant i<j \leqslant l, g_{n, i}$ and $h_{n, j}$ commute, while if $1 \leqslant j \leqslant i \leqslant l, d\left(\left[g_{n, i}, h_{n, j}\right] ; e_{G_{n}}\right) \geqslant \gamma$. Then under the failure of $\mathrm{CH}$, there are $2^{\aleph_{c}}$ many pairwise non isometrically isomorphic metric ultraproducts of the sequence $\left(G_{n_{k}}\right)_{k \in \mathbb{N}}$.

Lupini used this proposition for $\left(S_{n}, d_{\text {Hamm }}\right)_{n}$ to show that there are $2^{\aleph_{c}}$ many universal sofic groups. We shall use the same permutation that he constructed, regarded now as elements in $\left(G L_{n}(\mathbb{C}), d_{r k}\right)_{n}$ to show that the hypothesis of the proposition still holds for these groups.

Proposition 10.2. The hypothesis of Proposition 10.1 holds for $G L_{n}(\mathbb{C})$ endowed with the bi-invariant metric $d_{r k}$. The constant $\gamma$ can be chosen $2 / 9$.

Proof. As $\left(G L_{n}(\mathbb{C}), d_{r k}\right)_{n}$ are bi-invariant metric groups with uniformly bounded diameter we just need to construct elements $g_{n, i}$ and $h_{n, i}$. 
First assume that $n=3^{l}$ for some $l \in \mathbb{N}$. Let (12) and (23) be two transpositions in $S_{3}$ and denote by $A_{(12)}$ and $A_{(23)}$ the corresponding permutation matrices in $G L_{3}(\mathbb{C})$. For $1 \leqslant i \leqslant l$ define $g_{n, i}=A_{(12)} \otimes A_{(12)} \otimes \ldots \otimes A_{(12)} \otimes I d_{3^{l-i}}\left(A_{(12)}\right.$ is used $i$ times $)$ and $h_{n, i}=I d_{3^{i-1}} \otimes A_{(23)} \otimes I d_{3^{l-i}}$. It is easy to check that for $i<j g_{n, i}$ and $h_{n, j}$ commutes, while for $i \geqslant j\left[g_{n, i}, h_{n, j}\right]=I d_{3^{j-1}} \otimes A_{(123)} \otimes I d_{3^{l-j}}$. This means that $\left[g_{n, i}, h_{n, j}\right]$ is composed of $3^{l-1}$ cycles of length 3 , so $d_{r k}\left(\left[g_{n, i}, h_{n, j}\right], I d_{3^{l}}\right)=1-3^{l-1} / 3^{l}=2 / 3$.

Let now $n \in \mathbb{N}$ be an arbitrary number and $l \in \mathbb{N}$ such that $3^{l} \leqslant n<3^{l+1}$. Define $g_{n, i}=g_{3^{l}, i} \oplus I d_{n-3^{l}}$ and $h_{n, i}=h_{3^{l}, i} \oplus I d_{n-3^{l}}$. Again for $i<j g_{n, i}$ and $h_{n, j}$ commutes, while for $i \geqslant j\left[g_{n, i}, h_{n, j}\right]=I d_{3^{j-1}} \otimes A_{(123)} \otimes I d_{3^{l-j}} \oplus I d_{n-3^{l}}$. Then $d_{r k}\left(\left[g_{n, i}, h_{n, j}\right], I d_{3^{l}}\right)=1-\left(3^{l-1}+n-3^{l}\right) / n=\left(3^{l}-3^{l-1}\right) / n \geqslant 2 / 9$. Thus, the constant $\gamma$ can be set $2 / 9$.

\section{Almost Finite Dimensional REPRESENTATIONS}

In this section, we work only with unital algebras. The following propery of algebras was introduced by Gabor Elek.

Definition 11.1. ([El05, Definition 1.1]) A unital $F$-algebra $A$ has almost finite dimensional representations if for any finite dimensional subspace $1 \in L \subset A$ and $\varepsilon>0$, there exists a finite dimensional vector space $V$ together with a subspace $V_{\varepsilon} \subset V$ such that

(1) there exists a linear (not necessarily injective) map $\psi_{L, \varepsilon}: L \rightarrow \operatorname{End}_{F}(V)$ such that $\psi_{L, \varepsilon}(1)=I d$ and $\psi_{L, \varepsilon}(a) \psi_{L, \varepsilon}(b)(v)=\psi_{L, \varepsilon}(a b)(v)$ for $a, b, a b \in L$ and $v \in V_{\varepsilon}$.

(2) $\operatorname{dim}_{F} V-\operatorname{dim}_{F} V_{\varepsilon}<\varepsilon \cdot \operatorname{dim}_{F} V$.

Such a map is called an $\varepsilon$-almost representation of $L$.

Proposition 11.2. A unital algebra $A$ has almost finite dimensional representations if and only if there exists a unital morphism (not necessarily injective) $\Theta: A \rightarrow$ $\Pi M_{n_{k}}(F) / K \operatorname{Ker} \rho_{\omega}$.

Proof. Let $\left(L_{k}\right)_{k}$ be an increasing sequence of finite dimensional subspaces of $A$ such that $A=\bigcup_{k} L_{k}$ and $1 \in L_{k}$. Let $\left(\varepsilon_{k}\right)_{k}$ be a decreasing sequence of strictly positive reals such that $\lim _{k} \varepsilon_{k}=0$. For every $k$, let $\psi_{L_{k}, \varepsilon_{k}}: L_{k} \rightarrow \operatorname{End}_{F}\left(V_{k}\right)$ be the map from the previous definition. Define $n_{k}=\operatorname{dim}_{F} V_{k}$. Then $\Theta: A \rightarrow \Pi M_{n_{k}}(F) / K \operatorname{Ker} \rho_{\omega}$ defined by $\Theta=\Pi \psi_{L_{k}, \varepsilon_{k}} / \operatorname{Ker} \rho_{\omega}$ is a unital morphism. The reverse implication follows from the definition of ultraproduct. 
If we compare this definition to the definition of linear sofic algebras we see that having almost finite dimensional representations is the first step towards linear soficity. However, this is not sufficient. We introduce an object that measures how far from being linear sofic is an algebra with almost finite dimensional representations. This is inspired by the definition of the rank radical by Elek.

\subsection{The rank radical.}

Definition 11.3. ([E105, Definition 4.1]) The rank radical $R R(A)$ of an algebra is defined as follows: if $A$ does not have almost finite dimensional representations then $R R(A)=A$. Otherwise, let $p \in R R(A)$ if there exists a finite dimensional subspace $L$ with $\{1, p\} \subset L \subset A$ such that for any $\delta>0$ there exists $n_{\delta}>0$ with the following property: if $0<\varepsilon<n_{\delta}$ and $\psi_{L, \varepsilon}: L \rightarrow \operatorname{End}(V)$ is an $\varepsilon$-almost representation then $\operatorname{dim} \operatorname{Ran}\left(\psi_{L, \varepsilon}(p)\right)<\delta \cdot \operatorname{dim} V$.

We restate this property in ultraproduct language. We use the following definition.

Definition 11.4. If $1 \in L \subset A$ is a linear subspace of an algebra, then a partial morphism of $L$ is a linear function $\Phi: L \rightarrow \Pi M_{n_{k}}(F) / \operatorname{Ker} \rho_{\omega}$ such that $\Phi(1)=1$ and $\Phi(x) \Phi(y)=\Phi(x y)$ whenever $x, y, x y \in L$.

Proposition 11.5. For any element $p$ of an algebra $p \in R R(A)$ if and only if there exists a finite dimensional subspace $L$ with $\{1, p\} \subset L \subset A$ such that for any partial morphism $\Phi: L \rightarrow \Pi M_{n_{k}}(F) / \operatorname{Ker} \rho_{\omega}$ we have $\Phi(p)=0$.

Proof. We first assume that $A$ has almost finite dimensional representations. Note that this is equivalent to $R R(A) \varsubsetneqq A$.

Let $p \in R R(A)$. Let $L$ be the finite dimensional subspace from the definition of the rank radical. Fix $\delta>0$ and use again the definition to get a $n_{\delta}>0$. Let $\Phi: L \rightarrow \Pi M_{n_{k}}(F) / K \operatorname{Ker} \rho_{\omega}$ be a partial morphism, $\Phi=\Pi \phi_{k} / K \operatorname{Ker} \rho_{\omega}$. Let $0<\varepsilon<n_{\delta}$. Then there exists $H \in \omega$ such that $\phi_{k}: L \rightarrow M_{n_{k}}(F)$ is an $\varepsilon$-almost representation of $L$ for any $k \in H$. Then $\operatorname{dim} \operatorname{Ran}\left(\phi_{k}(p)\right)<\delta n_{k}$, or with our notation $\rho\left(\phi_{k}(p)\right)<\delta$ for any $k \in H$. This implies that $\rho_{\omega}(\Phi(p))<\delta$. As $\delta$ was arbitrary it follows that $\rho_{\omega}(\Phi(p))=0$ so $\Phi(p)=0$.

Suppose now $p \notin R R(A)$. Let $L$ be an arbitrary finite dimensional subspace. Then there exists $\delta_{L}>0$ such that for any $\varepsilon>0$ there exists an $\varepsilon$-almost representation 
$\psi_{L, \varepsilon}: L \rightarrow \operatorname{End}(V)$ with $\operatorname{dim} \operatorname{Ran}\left(\psi_{L, \varepsilon}(p)\right) \geqslant \delta_{L} \cdot \operatorname{dim} V$. This is equivalent to $\rho\left(\psi_{L, \varepsilon}(p)\right) \geqslant \delta_{L}$.

Let $\left(\varepsilon_{k}\right)_{k}$ be a decreasing sequence converging to 0 and $\psi_{L, \varepsilon_{k}}: L \rightarrow \operatorname{End}\left(V_{k}\right)$ be $\varepsilon_{k^{-}}$ almost representations with $\operatorname{dim} \rho\left(\psi_{L, \varepsilon_{k}}(p)\right) \geqslant \delta_{L}$. Define $\Psi=\Pi \psi_{L, \varepsilon_{k}} / \operatorname{Ker} \rho_{\omega}$. Because $\varepsilon_{k} \rightarrow 0, \Psi$ is a partial morphism of $L$. Also $\rho_{\omega}(\Psi(p)) \geqslant \delta_{L}$ so $\Psi(p) \neq 0$.

Consider now the case $R R(A)=A$. Let $p \in A$. We shall prove that there exists a finite dimensional subspace $L$ with $\{1, p\} \subset L \subset A$ such that there is no partial morphism $\Phi: L \rightarrow \Pi M_{n_{k}}(F) / K \operatorname{Ker} \rho_{\omega}$.

Let $A=\bigcup_{i} L_{i}$ where $\left(L_{i}\right)_{i}$ is an increasing sequence of finite dimensional subspaces of $A$ such that $\{1, p\} \in L_{i}$. Assume that for each $i$ there exists a partial morphism $\Phi_{i}: L_{i} \rightarrow \Pi M_{n_{i, k}}(F) / \operatorname{Ker} \rho_{\omega}$. Consider also $\left(\varepsilon_{i}\right)_{i}$ a sequence of strictly positive real numbers converging to 0 .

The existence of $\Phi_{i}$ implies the existence of $\psi_{i}: L_{i} \rightarrow M_{n_{i}, k_{i}}$ an $\varepsilon_{i}$-almost representation of $L_{i}$. Define $\Theta=\Pi \psi_{i} / \operatorname{Ker} \rho_{\omega}$. Because $A=\bigcup_{i} L_{i}$ and $\varepsilon_{i} \rightarrow 0, \Theta$ is a unital morphism. This is in contradiction with the fact that $A$ does not have almost finite dimensional representations.

Elek proved that $R R(A)$ is an ideal. We can deduce this from our description.

Corollary 11.6. The set $R R(A)$ is an ideal.

Proof. Let $a \in A$ and $p \in R R(A)$. Let $\{1, p\} \subset L_{p} \subset A$ be a finite dimensional subspace such that $\Phi(p)=0$ for any partial morphism $\Phi: L_{p} \rightarrow \Pi M_{n_{k}}(F) / K e r \rho_{\omega}$.

Define $L_{a p}=S p\left\{L_{p} \cup\{a, a p\}\right\}$, defined by thaking the linear span. Let $\Psi: L_{a p} \rightarrow$ $\Pi M_{n_{k}}(F) / \operatorname{Ker} \rho_{\omega}$ be a partial morphism. Then $\Psi(a p)=\Psi(a) \Psi(p)$. But $L_{p} \subset L_{a p}$ and $p \in R R(A)$ implies $\Psi(p)=0$. So $\Psi(a p)=0$. The same proof works for $p a$.

Theorem 11.7. The rank radical of $A / R R(A)$ is 0 .

Proof. We denote by $f: A \rightarrow A / R R(A)$ the canonical projection. Let $v \in A / R R(A)$, $v \neq 0$. Let $\{1, v\} \subset L$ be a finite dimensional subspace of $A / R R(A)$. Choose $N \subset A$ a finite dimensional subspace such that $f(N)=L$ and $1 \in N$. There exists $u \in N$ such that $v=f(u)$ and $u \notin R R(A)$. Define $N_{0}=S p\left\{N \cup N^{2}\right\} \cap R R(A)$. Then $N_{0}$ is finite dimensional and choose $\left\{z_{1}, \ldots, z_{r}\right\}$ a base for $N_{0}$. For any $1 \leqslant i \leqslant r$ there exists $L_{i} \subset A$ a finite dimensional subspace such that for any partial morphism $\Phi: L_{i} \rightarrow \Pi M_{n_{k}} / \operatorname{Ker} \rho_{\omega}$ $\Phi\left(z_{i}\right)=0$. 
Define $N_{1}=S p\left\{N \cup N^{2} \cup \bigcup_{i} L_{i}\right\}$. Because $u \notin R R(A)$ there exists a partial morphism $\Phi: N_{1} \rightarrow \Pi M_{n_{k}} / \operatorname{Ker} \rho_{\omega}$ such that $\Phi(u) \neq 0$. As $L_{i} \subset N_{1}$ we get $\Phi\left(z_{i}\right)=0$ for any $i$. This implies that $\Phi\left(N_{0}\right)=0$ so we can factor $\Phi$ to get a linear function $\Psi: L \cup L^{2} \rightarrow \Pi M_{n_{k}} / K \operatorname{Ker} \rho_{\omega}$. If $a, b \in L$ then $\Psi(a) \Psi(b)=\Psi(a b)$ so $\Psi$ restricted to $L$ is a partial morphism. Also $\Psi(v)=\Phi(u) \neq 0$. It follows that $v \notin R R(A / R R(A))$.

Proposition 11.8. ([E105, Proposition 4.3]) Let $A$ be an algebra such that $R R(A)=0$. Then $A$ is stably finite.

Proof. We simplify the original proof by the use of ultrafilters. First we prove that if $R R(A)=0$ then $R R\left(M_{m}(A)\right)=0$. Recall that $M_{m}(A) \simeq M_{m}(F) \otimes A$. Let $v \in M_{m}(A)$, $v \neq 0$ and let $u \in A$ be a nonzero entry of $v$. Consider $\{1, v\} \subset L \subset M_{m}(A)$ a finite dimensional subspace. Then there exists $\{1, u\} \subset L_{1} \subset A$ a finite dimensional subspace such that $L \subset M_{m}(F) \otimes L_{1}$. As $u \notin R R(A)$ there exists $\Phi_{1}: L_{1} \rightarrow \Pi M_{n_{k}}(F) / K \operatorname{Ker} \rho_{\omega}$ such that $\Phi(u) \neq 0$. Define $\Phi: M_{m}(F) \otimes L_{1} \rightarrow \Pi M_{m \cdot n_{k}}(F) / \operatorname{Ker} \rho_{\omega}$ by $\Phi(a \otimes p)=$ $a \otimes \Phi_{1}(p)$. Then $\Phi(v) \neq 0$.

Consider now $x, y \in A$ such that $x y=1$. Assume that $y x \neq 1$ so $x y-y x \neq 0$. Let $L=S p\{1, x, y, y x\}$. As $x y-y x \notin R R(A)$ there exists a partial morphism $\Phi: L \rightarrow \Pi M_{n_{k}}(F) / \operatorname{Ker} \rho_{\omega}$ such that $\Phi(x y-y x) \neq 0$. Now $1=\Phi(x y)=\Phi(x) \Phi(y)$ and by Proposition 2.8, $\Pi M_{n_{k}}(F) / \operatorname{Ker} \rho_{\omega}$ is directly finite. Thus, $\Phi(y) \Phi(x)=1$. It follows that $\Phi(x y-y x)=1-1=0$ contradiction.

11.2. The sofic radical. It is easy to see that if an algebra $A$ is linear sofic then $R R(A)=0$. However, this condition is not sufficient. We modify the definition of the rank radical to get a larger ideal that will describe linear soficity. This can be done also for groups and we first discuss this case as a warm up.

Definition 11.9. The linear sofic radical $L S R(G)$ of a group $G$ is defined as follows: $h \in L S R(G)$ whenever for all group morphisms $\Theta: G \rightarrow \Pi G L_{n_{k}}(\mathbb{C}) / d_{\omega}$ we have $\Theta(h)=1$.

Proposition 11.10. The linear sofic radical $L S R(G)$ is a normal subgroup of $G$. The group $G / L S R(G)$ is linear sofic.

Proof. It it easy to see from the definition that:

$$
L S R(G)=\bigcap\left\{\operatorname{Ker} \Theta \mid \Theta: G \rightarrow \Pi G L_{n_{k}}(\mathbb{C}) / d_{\omega} \text { group morphism }\right\},
$$


so indeed $L S R(G)$ is a normal subgroup of $G$.

For the second part of the proposition, for each $g \in G, g \notin L S R(G)$ consider a morphism $\Theta_{g}: G \rightarrow \Pi G L_{n_{k}}(\mathbb{C}) / d_{\omega}$ such that $\Theta_{g}(g) \neq 1$. Then $\left\{\widetilde{\Theta}_{g}\right\}_{g \in G \backslash L R S(G)}$ is a sequence of unital morphisms of the group algebra and we apply Proposition 7.6 to get a morphism $\Theta: G \rightarrow \Pi G L_{m_{k}}(\mathbb{C}) / d_{\omega}$ such that $\operatorname{Ker} \Theta=\bigcap_{g \in G \backslash L R S(G)} \operatorname{Ker} \Theta_{g}=$ $L R S(G)$.

This construction of linear sofic radical can be performed for several other metric approximation properties for groups, like soficity, weak soficity or hyperlinearity. We now introduce the sofic radical for algebras.

Definition 11.11. The sofic radical $S R(A)$ of an algebra is defined as follows: if $A$ does not have almost finite dimensional representations then $S R(A)=A$. Otherwise, let $p \in S R(A)$ if for any $\delta>0$ there exists a finite dimensional subspace $L$ with $\{1, p\} \subset L \subset A$ and there exists $n_{\delta}>0$ with the following property: if $0<\varepsilon<n_{\delta}$ and $\psi_{L, \varepsilon}: L \rightarrow \operatorname{End}(V)$ is an $\varepsilon$-almost representation then $\operatorname{dim} \operatorname{Ran}\left(\psi_{L, \varepsilon}(p)\right)<\delta \cdot \operatorname{dim} V$.

We now provide a characterization of the sofic radical in terms of morphisms into ultraproducts.

Proposition 11.12. For any element $p$ of an algebra $p \in S R(A)$ if and only if for any unital morphism $\Theta: A \rightarrow \Pi M_{n_{k}}(F) / \operatorname{Ker} \rho_{\omega}$ we have $\Theta(p)=0$.

Proof. Let $p \in S R(A)$. Fix $\delta>0$ and let $L$ and $n_{\delta}>0$ as in the definition of the sofic radical. Let $\Theta: A \rightarrow \Pi M_{n_{k}}(F) / K \operatorname{Ker} \rho_{\omega}$ be a unital morphism, $\Theta=\Pi \theta_{k} / K \operatorname{Ker} \rho_{\omega}$. Let $0<\varepsilon<n_{\delta}$. Then there exists $H \in \omega$ such that $\theta_{k}: L \rightarrow M_{n_{k}}(F)$ is an $\varepsilon$-almost representation of $L$ for any $k \in H$. Then $\operatorname{dim} \operatorname{Ran}\left(\theta_{k}(p)\right)<\delta n_{k}$, or with our notation $\rho\left(\theta_{k}(p)\right)<\delta$ for any $k \in H$. This implies that $\rho_{\omega}(\Theta(p))<\delta$. As $\delta$ was arbitrary it follows that $\rho_{\omega}(\Theta(p))=0$ so $\Theta(p)=0$.

Suppose now $p \notin S R(A)$. Then there exists $\delta>0$ such that for any finite dimensional subspace $L$ with $\{1, p\} \subset L \subset A$ and any $n>0$ there exists $0<\varepsilon<n$ and $\psi_{L, \varepsilon}: L \rightarrow \operatorname{End}(V)$ an $\varepsilon$-almost representation with $\operatorname{dim} \operatorname{Ran}\left(\psi_{L, \varepsilon}(p)\right) \geqslant \delta \cdot \operatorname{dim} V$. This is equivalent to $\rho\left(\psi_{L, \varepsilon}(p)\right) \geqslant \delta$.

Let $A=\bigcup_{k} L_{k}$ where $\left(L_{k}\right)_{k}$ is an increasing sequence of finite dimensional subspaces of $A$ such that $\{1, p\} \in L_{k}$. We can find a decreasing sequence $\left(\varepsilon_{k}\right)_{k}$ converging to 0 and $\varepsilon_{k}$-almost representations $\psi_{L_{k}, \varepsilon_{k}}: L_{k} \rightarrow \operatorname{End}\left(V_{k}\right)$ with $\operatorname{dim} \rho\left(\psi_{L_{k}, \varepsilon_{k}}(p)\right) \geqslant \delta$. 
Define $\Theta=\Pi \psi_{L_{k}, \varepsilon_{k}} / \operatorname{Ker} \rho_{\omega}$. Since $A=\cup_{k} L_{k}$ and $\varepsilon_{k} \rightarrow 0, \Theta$ is a unital morphism. Also $\rho_{\omega}(\Theta(p)) \geqslant \delta$ so $\Theta(p) \neq 0$.

Corollary 11.13. The sofic radical is an ideal. Moreover, $S R(A / S R(A))=0$.

Proof. By the previous proposition,

$$
S R(A)=\bigcap\left\{\operatorname{Ker} \Theta \mid \Theta: A \rightarrow \Pi M_{n_{k}}(F) / \operatorname{Ker} \rho_{\omega}, \text { unital morphism }\right\}
$$

Let now $q \in A / S R(A)$ and $p \in A$ such that $q=\hat{p}$. Suppose that $q \neq 0$ so that $p \notin S R(A)$. Then there exists a unital morphism $\Theta: A \rightarrow \Pi M_{n_{k}}(F) / \operatorname{Ker} \rho_{\omega}$ such that $\Theta(p) \neq 0$. Also $S R(A) \subset K \operatorname{Ker} \Theta$. So we can factor $\Theta$ by $S R(A)$ to get a unital morphism $\widetilde{\Theta}: A / S R(A) \rightarrow \Pi M_{n_{k}}(F) / \operatorname{Ker} \rho_{\omega}$ with $\widetilde{\Theta}(q) \neq 0$. It follows that $q \notin S R(A / S R(A))$.

Theorem 11.14. An algebra $A$ is linear sofic if and only if $S R(A)=0$.

Proof. If $A$ is linear sofic then there exists an injective morphism $\Theta: A \rightarrow$ $\Pi M_{n_{k}}(F) / \operatorname{Ker} \rho_{\omega}$. So $\operatorname{Ker} \Theta=0$ and then $S R(A)=0$.

Let $A$ be an algebra such that $S R(A)=0$. It follows that for any $p \neq 0$ there exists a unital morphism $\Psi_{p}: A \rightarrow \Pi M_{n_{k, p}}(F) / \operatorname{Ker} \rho_{\omega}$ such that $\Psi_{p}(p) \neq 0$.

Let $\left\{x_{i}\right\}_{i \in \mathbb{N}}$ be a basis for $A$ as a vector space over $F$. For $s \in \mathbb{N}$, we shall inductively construct $\Phi_{s}: A \rightarrow \Pi M_{n_{k, s}}(F) / \operatorname{Ker} \rho_{\omega}$ such that $\operatorname{Ker} \Phi_{s} \cap S p\left\{x_{1}, \ldots, x_{s}\right\}=0$.

Define $\Phi_{1}=\Psi_{x_{1}}$. Assume now by induction that we have $\Phi_{s-1}$ unital morphism such that $\operatorname{Ker} \Phi_{s-1} \cap \operatorname{Sp}\left\{x_{1}, \ldots, x_{s-1}\right\}=0$. Then $\operatorname{dim}\left(\operatorname{Ker} \Phi_{s-1} \cap \operatorname{Sp}\left\{x_{1}, \ldots, x_{s}\right\}\right) \leqslant 1$. If this space is trivial define $\Phi_{s}=\Phi_{s-1}$. Otherwise, let $y_{s} \in \operatorname{Ker} \Phi_{s-1} \cap \operatorname{Sp}\left\{x_{1}, \ldots, x_{s}\right\}$, $y_{s} \neq 0$ and define $\Phi_{s}=\Phi_{s-1} \oplus \Psi_{y_{s}}$ (see Proposition 7.3). If $z \in \operatorname{Ker} \Phi_{s} \cap \operatorname{Sp}\left\{x_{1}, \ldots, x_{s}\right\}$ then $z \in \operatorname{Ker} \Phi_{s-1} \cap \operatorname{Sp}\left\{x_{1}, \ldots, x_{s}\right\}$. It follows that $z \in \operatorname{Sp}\left\{y_{s}\right\}$. But also $z \in \operatorname{Ker} \Psi_{y_{s}}$ so $z=0$.

Using arguments similar to the proof of Theorem 7.4 (see also Proposition 7.6), we shall construct a unital morphism $\Theta$ such that $\operatorname{Ker} \Theta=\cap_{s} \operatorname{Ker} \Phi_{s}$.

First we bring $\Phi_{s}$ into the same sequence of matrix dimensions. Define $n_{k}=$ $n_{k, 1} n_{k, 2} \ldots n_{k, k}$. Replace $\Phi_{s}$ by an amplification to get $\Phi_{s}: A \rightarrow \Pi M_{n_{k}}(F) / \operatorname{Ker} \rho_{\omega}$.

Let now $\phi_{s, k}: A \rightarrow M_{n_{k}}$ be such that $\Phi_{s}=\Pi \phi_{s, k} / \operatorname{Ker} \rho_{\omega}$. Define $\theta_{k}: A \rightarrow M_{2^{k} n_{k}}$ by:

$$
\theta_{k}=\left(\phi_{1, k} \otimes I d_{2^{k-1}}\right) \oplus\left(\phi_{2, k} \otimes I d_{2^{k-2}}\right) \oplus \ldots \oplus\left(\phi_{k, k} \otimes I d_{2^{0}}\right) \oplus I d_{n_{k}}
$$


and $\Theta=\Pi \theta_{k} / \operatorname{Ker} \rho_{\omega}$. As in the proof of Theorem 7.4 $\rho_{\omega}(\Theta(p))=\sum_{s=1}^{\infty} \frac{1}{2^{s}} \rho_{\omega}\left(\Phi_{s}(p)\right)$. It follows that indeed $\operatorname{Ker} \Theta=\bigcap_{s} \operatorname{Ker} \Phi_{s}$. As $A=\bigcup_{s} S p\left\{x_{1}, \ldots, x_{s}\right\}$ and $\operatorname{Ker} \Phi_{s} \bigcap \operatorname{Sp}\left\{x_{1}, \ldots, x_{s}\right\}=0$ we get $\operatorname{Ker} \Theta=0$.

Corollary 11.15. A simple unital algebra with almost finite dimensional representations is linear sofic.

Proof. If $A$ has almost finite dimensional representations then $1 \notin S R(A)$. As $S R(A)$ is an ideal and $A$ is simple we get $S R(A)=0$. By the previous theorem $A$ is linear sofic.

Proposition 11.16. An amenable algebra without zero divisors is linear sofic.

Proof. Let $A$ be such an algebra. Consider $\left(L_{k}\right)_{k}$ an increasing sequence of finite dimensional subspaces of $A$ such that $1 \in L_{k}$ and $A=\bigcup_{k} L_{k}$. Consider also $\left(\varepsilon_{k}\right)_{k}$ a sequence of strictly positive real numbers such that $v_{k}=\operatorname{dim} L_{k} \cdot \varepsilon_{k} \rightarrow_{k} 0$. For any $k$, by the definition of amenability, there exists $S_{k}$ a finite dimensional subspace of $A$ such that $\operatorname{dim}\left(a S_{k} \cap S_{k}\right)>\left(1-\varepsilon_{k}\right) \cdot \operatorname{dim} S_{k}$ for any $a \in L_{k}$. Then we can construct a linear map $\phi_{k}(a): S_{k} \rightarrow S_{k}$ such that $\phi_{k}(a)$ is the left multiplication on a subspace of dimension $\left(1-\varepsilon_{k}\right) \cdot \operatorname{dim} S_{k}$. This implies that $\phi_{k}: L_{k} \rightarrow \operatorname{End}\left(S_{k}\right)$ is a $v_{k}$-almost representation of $L_{k}$. As $\phi_{k}(a)$ is the left multiplication on a subspace of dimension $\left(1-\varepsilon_{k}\right) \cdot \operatorname{dim} S_{k}$ and $A$ has no zero divisors it follows that $\rho\left(\phi_{k}(a)\right) \geqslant 1-\varepsilon_{k}$ for any $a \in L_{k}, a \neq 0$.

Let $n_{k}=\operatorname{dim} S_{k}$ and construct $\Theta: A \rightarrow \Pi M_{n_{k}} / \operatorname{Ker} \rho_{\omega}$ by $\Theta=\Pi \phi_{k} / \operatorname{Ker} \rho_{\omega}$. By construction $\rho_{\omega}(\Theta(a))=1$ for $a \neq 0$. It follows that $\Theta$ is injective, so $A$ is linear sofic.

The hypothesis of non-existence of zero divisors is too strong for this proposition to hold. We can construct a unital morphism $\Theta$ for any amenable algebra. Therefore, amenable algebras have almost finite dimensional representations. The non-existence of zero divisors implies $\rho_{\omega}(\Theta(a))=1$, but we only use $\rho_{\omega}(\Theta(a))>0$ for the injectivity of $\Theta$.

It is easy to construct almost finite dimensional representations for $L E F$ algebras (that is, algebras locally embeddable into finite dimensional ones [VG97, Zi02]) as also noticed in [E105]. In particular, any amenable or $L E F$ algebra that is also simple is linear sofic. 
There exist algebras that are not stably finite (see, for instance, Example 11.17 below). In particular, such algebras are not linear sofic. Combining Propositions 11.5 and 11.12 we immediately see that $R R(A) \subset S R(A)$. If $R R(A) \varsubsetneqq S R(A)$ then $A / R R(A)$ will be a stably finite non-linear sofic algebra. Such algebras seem difficult to find as counterexamples to soficity in general proved to be elusive.

Example 11.17. Let us present an example of an algebra that is directly finite but it is not stably finite. This construction is due to Sheperdson. Let $A$ be the unital algebra over $F$ generated by elements $\{x, y, z, t, a, b, c, d\}$ and relations $\{x a+y c=1 ; x b+y d=$ $0 ; z a+t c=0 ; z b+t d=1\}$. These relations are chosen such that:

$$
\left(\begin{array}{ll}
x & y \\
z & t
\end{array}\right)\left(\begin{array}{ll}
a & b \\
c & d
\end{array}\right)=I d_{2}
$$

Then $A$ is directly finite but it is not stably finite. Details can be found in [La07, Exercise 1.18, p. 11].

Example 11.18. In [Cor11] Cornulier constructed a sofic group that is not initially subamenable. Its group algebra is linear sofic by Theorem 1.2 and Proposition 4.5, On the other hand, this algebra is neither LEF by Theorem 1 of [Zi02], nor amenable.

11.3. Computations of the sofic radical. In this section, we prove that the rank radical is equal to the sofic radical for group algebras. We also provide a characterization of the sofic radical for a group algebra.

Proposition 11.19. Let $G$ be a countable group and let $L S R(G)$ be its linear sofic radical. Denote by $f: G \rightarrow G / L S R(G)$ the canonical projection and extend this morphism to group algebras: $\tilde{f}: \mathbb{C} G \rightarrow \mathbb{C}\left(G / L S R_{F}(G)\right)$. Then $S R(\mathbb{C} G)=\operatorname{Ker} \tilde{f}$.

Proof. Let $\Psi: \mathbb{C} G \rightarrow \Pi M_{n_{k}} / \operatorname{Ker} \rho_{\omega}$ be a unital morphism. By Proposition 2.6, $\Psi$ can be restricted to a morphism of the group, so $\Psi\left(u_{g}\right)=1$ if $g \in L S R(G)$. Then $f(g)=f(h)$ implies $\Psi\left(u_{g}\right)=\Psi\left(u_{h}\right)$. Now we can see that $\operatorname{Ker} \tilde{f} \subset \operatorname{Ker} \Psi$. As $\Psi$ was arbitrary, we get $\operatorname{Ker} \tilde{f} \subset S R(\mathbb{C} G)$.

The group $G / L S R(G)$ is linear sofic, so by Theorem 1.2 there exists $\Theta: \mathbb{C}(G / L S R(G)) \rightarrow \Pi M_{n_{k}} / \operatorname{Ker} \rho_{\omega}$ an injective unital morphism. Then $\Theta \circ \tilde{f}: \mathbb{C} G \rightarrow$ $\Pi M_{n_{k}} / \operatorname{Ker} \rho_{\omega}$ is a unital morphism such that $\operatorname{Ker} \Theta \circ \tilde{f}=\operatorname{Ker} \tilde{f}$. It follows that $S R(\mathbb{C} G) \subset K e r \tilde{f}$. 
Theorem 11.20. For any group $G$ we have $S R(\mathbb{C} G)=R R(\mathbb{C} G)$.

Proof. Let $p \notin R R(\mathbb{C} G)$ and assume that $p \in S R(\mathbb{C} G)$. Let $G=\bigcup_{i} B_{i}$ where $\left\{B_{i}\right\}_{i}$ is an increasing sequence of finite subsets each containing the support of $p$ such that $1 \in B_{i}$ and $B_{i}=B_{i}^{-1}$.

Let $\Phi_{i}: \mathbb{C}\left(B_{i} \cup B_{i}^{2}\right) \rightarrow \Pi_{k \rightarrow \omega} M_{n_{i, k}} / \operatorname{Ker} \rho_{\omega}$ be a partial morphism such that $\Phi_{i}(p) \neq$ 0 . Then $\Phi_{i}$ restricted to $B_{i}$ has its image included in $\prod_{k \rightarrow \omega} G L_{n_{i, k}} / d_{\omega}$. Now we can apply Proposition 5.12 to get a partial morphism $\Psi_{i}: \mathbb{C}\left(B_{i}\right) \rightarrow \prod_{k \rightarrow \omega} M_{m_{i, k}} / \operatorname{Ker} \rho_{\omega}$ such that for any $g, h \in B_{i}$ :

$$
\begin{aligned}
& \Phi_{i}\left(u_{g}\right)=\Phi_{i}\left(u_{h}\right) \Longrightarrow \Psi_{i}\left(u_{g}\right)=\Psi_{i}\left(u_{h}\right) \\
& \Phi_{i}\left(u_{g}\right) \neq \Phi_{i}\left(u_{h}\right) \Longrightarrow d_{\omega}\left(\Psi_{i}\left(u_{g}\right), \Psi_{i}\left(u_{h}\right)\right) \geqslant \frac{1}{4} .
\end{aligned}
$$

We construct the ultraproduct of the family $\left\{\Psi_{i}\right\}_{i}, \Psi=\prod_{i \rightarrow \omega} \Psi_{i} / d_{\omega}$. Then $\Psi$ : $\mathbb{C} G \rightarrow \Pi_{(i, k) \rightarrow \omega \otimes \omega} M_{m_{i, k}} / \operatorname{Ker} \rho_{\omega \otimes \omega}$ is a unital morphism. If $\Psi\left(u_{g}\right)=\Psi\left(u_{h}\right)$ then $\lim _{i \rightarrow \omega} d_{\omega}\left(\Psi_{i}\left(u_{g}\right), \Psi_{i}\left(u_{h}\right)\right)=0$. The properties of $\Psi_{i}$ imply that $\left\{i: \Psi_{i}\left(u_{g}\right)=\Psi_{i}\left(u_{h}\right)\right\} \in$ $\omega$ in this case.

Let $f: G \rightarrow G / L S R(G)$ be the canonical projection used also in the previous proposition. Then $f(g)=f(h)$ implies $\Psi\left(u_{g}\right)=\Psi\left(u_{h}\right)$. As argued earlier $\Psi\left(u_{g}\right)=$ $\Psi\left(u_{h}\right)$ iff $\left\{i: \Psi_{i}\left(u_{g}\right)=\Psi_{i}\left(u_{h}\right)\right\} \in \omega$. Because the support of $p$ is finite we can find an $i_{0}$ such that: $g, h \in \operatorname{supp} p$ and $f(g)=f(h)$ implies $\Psi_{i_{0}}\left(u_{g}\right)=\Psi_{i_{0}}\left(u_{h}\right)$. Then also $\Phi_{i_{0}}\left(u_{g}\right)=\Phi_{i_{0}}\left(u_{h}\right)$.

By the previous proposition and our initial assumption that $p \in S R(\mathbb{C} G)$ we get $\tilde{f}(p)=0$. This implies that $\Phi_{i_{0}}(p)=0$, which is a contradiction.

\section{REFERENCES}

[B08] L. Bartholdi, On amenability of group algebras. I. Israel J. Math. 168 (2008), 153-165.

[BCR98] J. Bochnak, M. Coste, M.-F. Roy, Real algebraic geometry, Ergebnisse der Mathematik und ihrer Grenzgebiete (3), 36, Springer-Verlag, Berlin, 1998.

[CaPă12] V. Capraro and L. Păunescu, Product Between Ultrafilters and Applications to the Connes' Embedding Problem J.Oper.Theory, 68(1) (2012), 165-172.

[CS09] T. Ceccherini-Silberstein and A. Samet-Vaillant, Asymptotic invariants of finitely generated algebras. A generalization of Gromov's quasi-isometric viewpoint, Functional analysis. J. Math. Sci. (N. Y.) 156(1) (2009), 56-108.

[Cor11] Y. Cornulier, A sofic group away from amenable groups, Math. Ann. 350(2) (2011), 269-275. 
[Del78] P. Delsarte, Bilinear forms over a finite field, with applications to coding theory, Journal of Combinatorial Theory A, 25 (1978), 226-241.

[El03] G. Elek, The amenability of affine algebras J. Algebra 264(2) (2003), 469-478.

[E105] G. Elek, On algebras that have almost finite dimensional representations, J. Algebra, 4(2) (2005), 179-186.

[ElSz04] G. Elek and E. Szabo, Sofic groups and direct finiteness, J. Algebra, 280(3) (2004), 426-434.

[ElSz06] G. Elek and E. Szabo, On sofic groups, J. Group Theory, 9(2) (2006), 161-171.

[FaSh09] I. Farah and S. Shelah, A dichotomy for the number of ultrafilters, (2009), ARXIV:0912.0406.

[Gab85] E. Gabidulin, Theory of codes with maximum rank distance, Problems on Information Transmission, 21(1) (1985), 1-12.

[GIRi08] L. Glebsky and L. M. Rivera, Sofic groups and profinite topology on free groups, J. Algebra, 320(9) (2008), 3512-3518.

[GIRi09] L. Glebsky and L. M. Rivera, On low rank perturbations of complex matrices and some discrete metric spaces, Electron. J. Linear Algebra 18 (2009), 302-316.

[Gr99] M. Gromov, Endomorphism of symbolic algebraic varieties, J. Eur. Math. Soc., 1 (1999), 109-197.

[Gr08] M. Gromov, Entropy and isoperimetry for linear and non-linear group actions, Groups Geom. Dyn., 2(4) (2008), 499-593.

[Hua45] L.-K. Hua, Geometries of matrices. I. Generalizations of von Staudt's theorem, Trans. Amer. Math. Soc., 57 (1945), 441-481.

[IiIwa09] K. Iima and R. Iwamatsu, On the Jordan decomposition of tensored matrices of Jordan canonical forms, Math. J. Okayama Univ., 51 (2009), 133-148.

[La07] T. Lam, Exercise in Modules and Rings, Problem books in mathematics, Springer, 2007.

[Lu11] M. Lupini, Logic for metric structures and the number of universal sofic and hyperlinear groups, (2011), ARXIV:1111.0729, to appear in Proc. Amer. Math. Soc.

[Ma40] A. Malcev, On isomorphic matrix representations of infinite groups, Mat. Sb., 8(50) (1940), 405422.

[MaVl] A. Martsinkovsky and A. Vlassov, The representation rings of $k[x]$, preprint, http://www.math.neu.edu/martsinkovsky/GreenExcerpt.pdf.

[Oz09] N. Ozawa, Hyperlinearity, sofic groups and applications to group theory, (2009), http://www.kurims.kyoto-u.ac.jp/ narutaka/notes/NoteSofic.pdf.

[Pe08] V. Pestov, Hyperlinear and sofic groups: a brief guide, Bull. Symbolic Logic, 14(4) (2008), 449480.

[PeKw09] V. Pestov and A. Kwiatkowska, An introduction to hyperlinear and sofic groups, (2009), ARXIV:0911.4266.

[Ră08] F. Rădulescu, The von Neumann algebras of the non-residually finite Baumslag group $\langle a, b|$ $\left.a b^{3} a^{-1}=b^{2}\right\rangle$ embeds into $R^{\omega}$, Hot topics in operator theory, Theta Ser. Adv. Math., 9, Theta, Bucharest, (2008), 173-185. 
[S00] A. Samet-Vaillant, $C^{*}$-algebras, Gelfand-Kirillov dimension, and Følner sets J. Funct. Anal. 171(2) (2000), 346-365.

[S06] P. Šemrl, Maps on matrix and operator algebras, Jahresber. Deutsch. Math.-Verein., 108(2) (2006), 91-103.

[VG97] A. Vershik and E. Gordon, Groups that are locally embeddable in the class of finite groups, Algebra i Analiz 9(1) (1997), 71-97; translation in St. Petersburg Math. J. 9(1) (1998), 49-67.

[Wan96] Z.-X. Wan, Geometry of matrices, World Scientific, Singapore, (1996).

[W00] B. Weiss, Sofic groups and dynamical systems, Ergodic theory and harmonic analysis (Mumbai, 1999), Sankhyā Ser. A, 62(3) (2000), 350-359.

[Zi02] M. Ziman, On finite approximations of groups and algebras, Illinois J. of Math., 43(3) (2002), $837-839$.

(G. Arzhantseva) University of Vienna, Faculty of Mathematics, Nordbergstraße 15, 1090 WiEn, Austria

E-mail address: goulnara.arzhantseva@univie.ac.at

(L. Păunescu) University of Vienna, Faculty of Mathematics, Nordbergstraße 15, 1090 Wien, Austria And Institute of Mathematics of THE Romanian ACAdemy (ON LEAVE), 21 Calea Grivitei Street, 010702 Bucharest, Romania

E-mail address: liviu.paunescu@univie.ac.at 